\title{
Effects of Forward- and Backward-Facing Steps on the Crossflow Receptivity and Stability in Supersonic Boundary Layers
}

\author{
P. Balakumar, Rudolph A. King, and Jenna L. Eppink \\ Flow Physics and Control Branch \\ NASA Langley Research Center, Hampton, VA 23681
}

\begin{abstract}
The effects of forward- and backward-facing steps on the receptivity and stability of three-dimensional supersonic boundary layers over a swept wing with a blunt leading edge are numerically investigated for a freestream Mach number of 3 and a sweep angle of 30 degrees. The flow fields are obtained by solving the full Navier-Stokes equations. The evolution of instability waves generated by surface roughness is simulated with and without the forward- and backward-facing steps. The separation bubble lengths are about 5-10 step heights for the forward-facing step and are about 10 for the backward-facing step. The linear stability calculations show very strong instability in the separated region with a large frequency domain. The simulation results show that the presence of backward-facing steps decreases the amplitude of the stationary crossflow vortices with longer spanwise wavelengths by about fifty percent and the presence of forward-facing steps does not modify the amplitudes noticeably across the steps. The waves with the shorter wavelengths grow substantially downstream of the step in agreement with the linear stability prediction.
\end{abstract}

\section{Introduction}

Major technical and operational challenges exist in achieving and maintaining laminar flow over swept wings. ${ }^{1,2}$ The first technical challenge includes the design of a laminar wing that gives the maximum extent of laminar flow and provides the desired overall aerodynamic characteristics such as lift, drag and moments. The challenges in designing a laminar wing include the accurate prediction of the laminar-to-turbulent transition fronts and the ability to control the different boundary-layer instabilities that cause transition in these flows. These boundary-layer instabilities may include, but are not limited to, attachment-line, crossflow, and Tollmien-Schlichting (TS) instabilities. Boundary-layer transition over swept wings near the leading edge is typically caused by the crossflow instability induced by spanwise pressure gradients provided that the attachment-line instability is subcritical. ${ }^{3,4}$ Crossflow instability is comprised of both three-dimensional traveling and stationary disturbances. The stationary crossflow vortices originate from three-dimensional roughness elements and the transition onset is mainly determined by the roughness amplitude and distribution that exists near the leading edge of the wing. Transition also results from traveling disturbances that are generated by freestream acoustic and/or turbulent disturbances or by the interaction between freestream disturbances and surface roughness. In our previous papers, ${ }^{5,6}$ we investigated the receptivity of the stationary and traveling crossflow vortices to three-dimensional roughness elements and to freestream vortical and acoustic waves.

The second challenge is to achieve and maintain the surface imperfections in the expected laminar flow region below the threshold values so that large inhomogenities on the surface do not cause early transition. The imperfections may be realized during manufacturing and/or the normal daily operations of the aircraft. The imperfections include forward- and backward-facing steps, gaps, wavy bulges, and large three-dimensional roughness elements.

Fage $^{7}$ was the first to obtain empirical relations from wind tunnel experiments performed on a flat plate and on an airfoil with bulges, hollows and ridges to determine the minimum height of these imperfections that affect the position of boundary-layer transition. It was also recognized that the effects

1 of 27

American Institute of Aeronautics and Astronautics 
of these surfaces on transition are associated with the separation bubbles formed on the surfaces. Carmichael $^{8}$ developed a criterion based on flight and wind tunnel experiments for the allowable waviness (wave height / wavelength) that can be permitted without affecting transition for swept and unswept wings. The critical waviness depends on Reynolds number, Mach number, wing sweep, wavelength, wave location, and number of waves. Empirical criteria ${ }^{9}$ for the allowable steps and gaps were obtained from the X-21 flight experiment and from the NASA natural laminar flow (NLF) glove experiments. The established critical Reynolds numbers $\mathrm{R}_{\mathrm{h}, \text { crit }}=\left(U_{\infty} / v\right) h$, where $U_{\infty}$ is the freestream

velocity, $v$ the kinematic viscosity and h the height of the step or the length of the gap were, 900,1800 and 15,000 for backward-facing steps, forward-facing steps and gaps, respectively.

Klebanoff et al. ${ }^{10}$ performed detailed experimental investigations of the mechanisms involved in twodimensional roughness-induced transition in incompressible flows. The major findings were that twodimensional roughness elements caused early transition compared to that without roughness due to the destabilizing influence of the inflectional boundary-layer profiles behind the roughness. It was also demonstrated that the upstream movement of the transition location towards the roughness is gradual with increasing Reynolds number. These findings initiated the approach for the use of linear stability theory on roughness-induced transition, i.e., the effects of roughness on transition using linear stability analysis on the flow field induced by the roughness. Nayfeh et al. ${ }^{11}$ computed the stability characteristics of flows around humps and dips. The calculations showed small separation bubbles behind the humps when the height to width ratio exceeded a critical value. In these cases, the amplification rates of the disturbances were much larger downstream of the hump compared to the unperturbed case. The integrated growthrates, Gctors, had large jumps across the separation zone and remained parallel to the unperturbed case further downstream. Similar to the previous analysis, Massad and Iyer ${ }^{12}$ computed the stability characteristics and the N-Factors for flows behind two-dimensional humps up to high subsonic Mach numbers. The predicted transition onset locations based on an N-Factor of 9 correlated very well with the experimentally observed transition data of Fage ${ }^{7}$.

Instead of computing the stability properties across two-dimensional obstacles, semi-empirical methods ${ }^{13-21}$ were developed to estimate the expected increments in N-Factors, $\Delta \mathrm{N}$, across steps and gaps. The transition onset points were then determined using the N-Factor increments and computing the stability characteristics for the unperturbed boundary layer. Crouch et al. ${ }^{13}$ proposed a simple formula where $\Delta \mathrm{N}=1.6 \mathrm{~h} / \delta_{1}$ and $4.4 \mathrm{~h} / \delta_{1}$ based on experimental and computational analysis for the forward- and backward-facing steps, respectively. Here $\delta_{1}$ is the displacement thickness and $h$ the step height. Numerical and experimental investigations have been performed at ONERA ${ }^{14,15,16}$ to determine the effects of steps and gaps on boundary-layer transition over unswept and swept wings in incompressible and compressible flows. Stability computations were performed to estimate the N-Factor increments across the steps and gaps that were placed on a flat plate ${ }^{15}$. The mean flow computations over a backward-facing step showed a large separation bubble downstream of the step. The length of the separation bubble was about 30 times the height of the step. The N-Factor envelope curve showed a jump across the steps for both forward- and backward-facing steps. Downstream of the backward-facing step the N-Factor curve remained parallel to that of the unperturbed case. For the forward-facing step, the N-Factor curve approached that of the unperturbed case downstream. Experiments were performed ${ }^{15}$ on a constant chord ONERA model at three sweep angles and at two angles of attack. The measured transition fronts with forward-facing steps showed that up to a critical Reynolds number of $R_{h}=2000$, there was no noticeable movement in the transition location at zero sweep and at small angle of attack. Beyond this value, the transition point moved rapidly towards the step. When the sweep angle was increased to 50 degrees, the critical Reynolds number moved to 2500. At high angles of attack, the critical Reynolds number was about 2000 at zero sweep angle and it decreased to 1500 at 50-degrees sweep angle. For backward-facing steps, the movement of the transition point was gradual with increasing step height. The critical Reynolds numbers at small angle of attack was about 350 at zero sweep and increased to 500 at 50-degrees sweep. At high angles of attack, these numbers were 900 and 700 at zero and 50-degrees sweep angles. The influence of forward-facing steps on the growthrate of instability waves in transonic flows was

2 of 27

American Institute of Aeronautics and Astronautics 
investigated ${ }^{17}$ using linear stability theory and direct numerical simulation. The mean flow computations revealed that the length of the separation bubble was about 8 times the height of the step at the freestream Mach number of 0.8 and it was about 40 at a slightly supersonic Mach number of 1.06. Similar to the ONERA experiment for the subsonic case, the N-Factor envelope curve showed a jump across the step and the N-Factor increment approaches zero far downstream. In the slightly supersonic case, the N-Factor curve first increased upstream of the step and then decreased downstream of the step before it followed

parallel to the unperturbed case. The comparison with the existing semi-empirical methods ${ }^{13,16}$ showed poor comparison between the computed and the predicted $\Delta \mathrm{N}$ values.

Eppink $^{22}$ recently performed detailed measurements of the instabilities in the $3 \mathrm{D}$ flow over a backward-facing step. The experiment was performed at low speeds using a swept flat plate with a pressure body to create a stationary-crossflow dominated flow for the smooth-wall case. A step height of approximately $40 \%$ of the boundary-layer thickness resulted in a local increase in stationary crossflow amplitude just downstream of the reattachment location, which was approximately 30 step heights downstream of the step. The step was seen to have a larger effect on the growth of the smaller wavelength stationary crossflow mode compared to the larger (forced) wavelength, resulting in a maximum $\Delta \mathrm{N}$ of 1.8 compared to 0.8 for the larger wavelength. However, stationary crossflow was of relatively low amplitude at transition, and instead transition was dominated by a broad frequency band of unsteady disturbances resulting from the presence of the step. The lower frequency disturbances may have been traveling crossflow and/or TS-like disturbances, but it is unclear. The higher frequency disturbances were shear-layer instabilities.

In this paper we consider the effects of steps on the stationary and traveling crossflow instability waves. Knowing the allowable manufacturing tolerances for maintaining laminar flow is critical for the design and maintenance of a laminar wing. In all of the previous investigations to the authors' knowledge, the local receptivity due to steps and gaps has not been considered in three-dimensional boundary layers. Also, how the steps modify the incoming stationary and traveling crossflow vortices were also not investigated. Logically we expect the local imperfections to basically modify the incoming boundary layer. But how are the amplitude and growth of the incoming instability waves modified by the steps? And, are new instabilities introduced by the modified boundary layer? In this paper we investigate these two questions. We generate the stationary crossflow vortices by placing periodic roughness elements near the leading edge of the wing and perform the simulations with and without forward- and backward-facing steps located at different chordwise positions. The simulations are performed by solving the threedimensional Navier-Stokes equations using a $5^{\text {th }}$-order accurate weighted essentially non-oscillatory (WENO) scheme for space discretization and using a $3^{\text {rd }}$-order, total variation diminishing (TVD) RungeKutta scheme for time integration. Computations are performed for the supersonic flow over a swept wing at a freestream Mach number of 3 and at a sweep angle of 30 degrees. The reference configuration and test conditions are those in our previous papers ${ }^{5,6}$ and in Ref. 23.

\section{Formulation of the problem}

We consider supersonic flow over an infinite swept wing with a blunt leading edge (Fig. 1). The Cartesian coordinates, $(x, y, z)$, are oriented such that $x$ is along the chord direction perpendicular to the leading edge, $z$ is along the spanwise direction and $y$ is along the normal direction. The sweep angle is $\Lambda$, the freestream Mach number is $M$ and the freestream velocity is $q_{\infty}$. We are interested in the effects of forward- and backward-facing steps on transition dominated by stationary and traveling crossflow instabilities in three-dimensional supersonic boundary layers. The variables density $\rho$, pressure $p$ and velocity are nondimensionalized by the respective freestream values $\rho_{\infty}, p_{\infty}$ and $q_{\infty}$.

\section{A. Roughness}

We consider a three-dimensional, spanwise-periodic roughness strip placed on the surface of the wing very close to the leading-edge region near the neutral point. The shape of the roughness is in the form

3 of 27

American Institute of Aeronautics and Astronautics 


$$
y_{r}(x, z)=h_{r} e^{-\sigma_{r}\left(\frac{x-x_{r}}{\Delta x_{r}}\right)^{2}} \cos \beta z
$$

Here $y_{r}$ is the height of the roughness normal to the surface of the wing, $h_{r}$ is the maximum height, $x_{r}$ is the surface location of the roughness, $\Delta x_{r}$ is related to the spatial extent of the roughness along the wing chord, $\beta$ is the spanwise wave number, and $\sigma_{r}$ is a constant that determines the width of the roughness in the $x$-direction.

\section{B. Forward- and backward-facing steps}

Two-dimensional forward- and backward-facing steps are introduced near the leading edge of the wing. The shape of a step is in the form:

$$
y_{s}(x)= \pm \frac{h_{s}}{2}\left[\tanh \sigma_{s} \frac{x-x_{s}}{\Delta x_{s}}+1\right]
$$

Here $y_{s}(x)$ is the height of the step normal to the surface of the plate, $h_{s}$ is the maximum height, $x_{s}$ is the location of the center of the step, and $\sigma_{s}$ and $\Delta x_{s}$ determine the chordwise spatial extent of the step.

\section{Governing Equations}

The Governing equations and the solution algorithms are given in our previous paper ${ }^{6}$. The equations solved are the three-dimensional unsteady compressible Navier-Stokes equations in conservation form. The governing equations are solved using a $5^{\text {th }}$-order accurate weighted essentially non-oscillatory (WENO) scheme for space discretization and a $3^{\text {rd }}$-order total variation diminishing (TVD) Runge-Kutta scheme for time integration.

At the outflow boundary, extrapolation is used to obtain the flow variables. At the wall, no-slip conditions are used for the velocities and a constant temperature condition is employed for the temperature. The density at the wall is computed from the continuity equation. In the spanwise direction, periodic conditions are imposed at the boundaries. The freestream values are prescribed at the outer boundary that lies outside the bow shock and simulations are performed using a variable time step until the maximum residual reaches a small value on the order of $10^{-11}$. A CFL number of 0.4 is used in these computations.

We use a body-fitted curvilinear grid system in all of the simulations. The grid stretches in the wall normal $\eta$ direction close to the wall and is uniform outside the boundary layer. In the streamwise $\xi$ direction, the grid is symmetric about the leading edge, and is very fine near the leading edge and across the steps. The grid is uniform in the spanwise $\zeta$ direction. Figs. $1(c-d)$ show the grid system employed for the flow over a forward- and a backward-facing steps in the $(x, y)$ plane. We use 251 points in the normal direction, 41 points in the spanwise direction and about 1501 points in the chordwise direction.

\section{Results}

We consider a supersonic flow over an infinite swept wing with a blunt leading edge at zero angle of attack as presented Fig. 1. The airfoil is a biconvex shape with a constant curvature radius. The chord length is $150 \mathrm{~mm}$ and the maximum thickness is $30 \mathrm{~mm}$. A parabola with a leading-edge radius of $6 \mathrm{~mm}$ models the leading edge of the wing. The flow parameters and the dimensions are given in Table 1. The transition onset location in the experiment ${ }^{23}$ for the flow parameters in the table occurred at $x=45 \mathrm{~mm}$ 
from the leading edge. The computational domain extends from $x=-10$ to $60.0 \mathrm{~mm}$ in the axial direction. Detailed mean flow, receptivity, and stability results were given in the previous papers. ${ }^{5,6}$

\section{Table 1. Flow parameters for the swept wing model}

Chord length: $\mathrm{C}=150 \mathrm{~mm}$

Maximum thickness: $\mathrm{t}=30 \mathrm{~mm}$

Freestream Mach number: $M_{\infty}=3.0$

Sweep angle: $\Lambda=30$ degrees

Angle of attack: $\mathrm{AOA}=0$ degrees

Freestream Reynolds number: $\operatorname{Re}_{\infty}=18.0 \times 10^{6} / \mathrm{m}$.

Freestream temperature: $T_{\infty}=121.42 \mathrm{~K}$

Wall temperature: $T_{w}=300.0 \mathrm{~K}$

\section{(a) Without steps}

Figures $2(a, b)$ show the density contours and surface pressure coefficient obtained from the NavierStokes simulation. The normal Mach number is 2.60. Due to the blunt leading edge, the shock is detached from the nose a distance of $2.8 \mathrm{~mm}$. The pressure has a favorable distribution from the leading edge. Figures $3(a, b)$ display the velocity profiles along the inviscid streamlines and crossflow velocity profiles at different axial locations $(x=1,5,10,15$ and $40 \mathrm{~mm})$. The boundary-layer thicknesses at these locations are approximately $0.075,0.12,0.18,0.24$ and $0.50 \mathrm{~mm}$, respectively. The maximum crossflow velocity remains almost constant at a value of approximately 0.07 starting from the leading edge.

\section{(b) With forward- and backward-facing steps}

Computations were performed for forward- and backward-facing steps of varying heights located at different stations along the wing. The shape of the step is given in eq. (2) and depicted in Figs. 1(c) and (d). Table 2 gives the locations $x_{s}$ and heights $h_{s}$ of the steps for the simulations performed. The steps are located at $x_{s}=5,10$, and $15 \mathrm{~mm}$ from the leading edge of the wing. The heights of the steps at these locations are $h_{s}=0.08,0.12 \mathrm{~mm}$ at $x_{s}=5 \mathrm{~mm}, h_{s}=0.06,0.12$ and $0.18 \mathrm{~mm}$ at $x_{s}=10 \mathrm{~mm}$ and $h_{s}=0.08 \mathrm{~mm}$ at $x_{s}=15 \mathrm{~mm}$, respectively. We used a constant value for the parameter $\sigma_{s}=4$. The ratio of the step height to the boundary-layer thickness $\delta$ varies from $h_{s} / \delta=1 / 3$ to 1 . The Reynolds number based on the parameters at the step height $R e_{k, u}=|u|_{\mathrm{k}} h_{s} / v_{k}$ and the Reynolds number based on the boundary-layer edge values $\operatorname{Re}_{h}=|u|_{\mathrm{e}} h_{s} / v_{e}$ are given in Table 2 . Here $|u|$ is the velocity parallel to the surface along the chordwise direction.

Table 2. Step parameters

\begin{tabular}{cccccccc}
\hline$x_{s}(\mathrm{~mm})$ & Step & $h_{s}(\mathrm{~mm})$ & $\Delta x_{s}(\mathrm{~mm})$ & $h_{s} / \delta$ & $R e_{k, u}$ & $R e_{h}$ & $\Delta x_{\text {sep }} / h_{s}$ \\
\hline 5 & Forward & 0.08 & 0.04 & $2 / 3$ & 790 & 910 & 6.5 \\
5 & & 0.12 & 0.06 & 1 & 1370 & 1370 & 6.5 \\
10 & & 0.06 & 0.04 & $1 / 3$ & 300 & 687 & 5.0 \\
10 & & 0.12 & 0.06 & $2 / 3$ & 1150 & 1370 & 9.0 \\
10 & & 0.18 & 0.09 & 1 & 2060 & 2060 & 10.0 \\
15 & & 0.08 & 0.04 & $1 / 3$ & 385 & 907 & 7.5 \\
& & & 5 of 27 & & & &
\end{tabular}


$44^{\text {th }}$ AIAA Fluid Dynamics Conference, June 16-20, 2014, Atlanta, Georgia

\begin{tabular}{|c|c|c|c|c|c|c|}
\hline Backward & 0.08 & 0.04 & $2 / 3$ & 790 & 910 & 9.0 \\
\hline 5 & 0.12 & 0.06 & 1 & 1370 & 1370 & 9.0 \\
\hline 10 & 0.06 & 0.04 & $1 / 3$ & 300 & 687 & 9.0 \\
\hline 10 & 0.12 & 0.06 & $2 / 3$ & 1150 & 1370 & 10.0 \\
\hline 10 & 0.18 & 0.09 & 1 & 2060 & 2060 & 10.0 \\
\hline 15 & 0.08 & 0.04 & $1 / 3$ & 385 & 907 & 10.0 \\
\hline
\end{tabular}

We will present detailed results for the mean flow in this section for the case with the roughness located at $x_{s}=10 \mathrm{~mm}$ and the step height of $h_{s}=0.12 \mathrm{~mm}$. Figures $4(\mathrm{a}, \mathrm{b})$ and $5(\mathrm{a}, \mathrm{b})$ show the $u$-velocity contours and the streamlines for the flow over a forward- and a backward-facing step, respectively. As expected, the forward-facing step generates a weak shock and an expansion wave and creates a separation bubble upstream of the step. Similarly, the backward-facing step generates a weak expansion wave and a compression wave and induces a separation bubble downstream of the step. The flow separates at about 9.0 step heights upstream of the step for the forward-facing step, $\Delta x_{\text {sep }} / h_{s}=9.0$, and reattaches on the upper part of the step. Similarly for the backward-facing step, the flow separates from the top of the step and reattaches at about 10 step heights downstream, $\Delta x_{\text {sep }} / h_{s}=10$. The separation lengths (see Table 2) are comparatively low compared to the flow over steps in flat plate boundary layers. The computations with increasing heights show that the separation bubble lengths increase for forward-facing steps. For the case of $x_{s}=10 \mathrm{~mm}$, the separation lengths are $\Delta x_{\text {sep }} / h_{s}=5,9$ and 10 for $h_{s}=0.06,0.12$ and $0.18 \mathrm{~mm}$, respectively. However, the separation lengths behind the backward-facing steps remain almost constant at $\Delta x_{\text {sep }} / h_{s}=10$. The separation lengths in flows over backward-facing steps in flat plate boundary layers in incompressible flows ${ }^{15}$ are larger than $\Delta x_{\text {sep }} / h_{s}>30$. We have also performed unpublished simulations for flows over forward- and backward-facing steps in a flat plate supersonic flow at a Mach number of 2.0 and found that the separation lengths are in the range of 25 . However, experimental measurements by Chen et al. ${ }^{24}$ on a flat plate boundary layer at a Mach number of 3.0 indicate separation distances of $\Delta x_{\text {sep }} / h_{s}=7$ to 7.5 downstream of a backward-facing step where $h_{s} / \delta \sim 10$.

Figure 6 depicts the surface pressure distribution over the wing with and without the steps. The results are shown for the forward- and backward-facing steps of different heights located at $x_{s}=10$ and $15 \mathrm{~mm}$. For the forward-facing steps, the pressure starts to increase gradually well upstream of the separation point, about 20 step heights upstream, and peaks at the step location. The pressure drops immediately downstream of the step and merges with the unperturbed value. The pressure modifications are similar for all the heights, but the amplitude of the pressure increases with the step height. Similarly, for the backward-facing steps, the pressure drops immediately at the step and recovers to the unperturbed value within about 20 step heights.

Figures $7(a, b)$ show the computed boundary-layer profiles at several upstream and downstream stations for the forward- and backward-facing steps, respectively. Here $\Delta x=x-x_{s}$ is the distance measured from the step. The steps are located at $x_{s}=10 \mathrm{~mm}$ and the height is $h_{s}=0.12 \mathrm{~mm}$. We also included two profiles obtained without the steps at an upstream and a downstream station where the profiles with and without the steps are almost the same. For the forward-facing step, the boundary layer starts to deviate from the unperturbed case near the station $\Delta x=-25 h_{s}$. Further downstream the profiles become inflectional and the flow separates around $\Delta x=-9 h_{s}$. The boundary layers also become strongly inflectional. The maximum negative velocity in the separation bubble is about -0.017 . Far downstream $\Delta x=84 h_{s}$, the boundary layer recovers back to the unperturbed profile. Similarly, Fig. 7(b) depicts the results for the backward-facing step. The profiles starts to deviate from the unperturbed case close to $\Delta x=-8 h_{s}$. The profiles become fuller up to the step $\Delta x=-1 h_{s}$. Immediately downstream of the step a strong shear layer forms and the boundary layer becomes thicker. The maximum reverse velocity inside the separation bubble is about -0.056 , which is about 3 times larger than the value for the forward-step case. The flow reattaches close to $\Delta x=10 h_{s}$ and far downstream $\left(\Delta x=84 h_{s}\right)$ it recovers back to the unperturbed case. It is

6 of 27

American Institute of Aeronautics and Astronautics 
also seen that the shear layer velocity gradient downstream of the backward-facing step is much stronger than that in the forward-facing step case. This will have implications in the stability characteristics of flows over forward- and backward-facing steps. Similarly, Figures 8(a) and (b) depict the results for the step location $x_{s}=15 \mathrm{~mm}$ with the step height of $h_{s}=0.08 \mathrm{~mm}$. The general conclusions are the same as in the previous case. The maximum velocities in the separation zones are -0.004 and -0.035 for the forwardand backward-facing steps.

\section{B. Linear instability}

\section{(a) Without the steps}

Figures $9(a, b)$ show the stability characteristics of the boundary layer for the smooth case. Computations were performed for constant spanwise wavelengths and included curvature effects. Figure 9(a) shows the variation of the growthrate as a function of frequency for different spanwise wavelengths at the station $x=20 \mathrm{~mm}$. We also included the variation of the waveangle for one spanwise wave number of $\lambda_{z}=1.25 \mathrm{~mm}$. The waveangle is measured from the inviscid streamline in the anticlockwise direction as illustrated in Fig. 10. Without the crossflow velocity component, the stability characteristics are symmetric in waveangle with respect to the inviscid streamline. In two-dimensional supersonic boundary layers, the most amplified waves are oblique, inclined between 45 to 70 degrees from the inviscid streamlines $^{25}$. In three-dimensional boundary layers, crossflow velocity introduces a new instability. These crossflow instability waves are confined between 80 to 100 degrees from the inviscid streamlines. These different instability waves are schematically illustrated in Fig. 10. When the waveangle increases from the inviscid streamline in the anticlockwise direction, the first mode becomes unstable in region 3. With further increase in waveangle, the crossflow instability becomes unstable in region 2 . This region is narrowly confined to a small wedge in the direction opposite to the crossflow velocity component. The stationary crossflow wave vector is inclined very close to the direction opposite to the crossflow velocity. With further increase in waveangle the first mode becomes unstable again in region 1 . The maximum growth occurs for the waves that lie to the right of the stationary crossflow direction (i.e. e. towards the first mode 3 region from the stationary crossflow direction). If we confine the analysis only to the positive streamwise direction, region 3 is the same as region 4 shown in the opposite quadrant. The associated wave propagation directions are also shown as arrows in this figure.

Figure 9(a) depicts a typical growthrate and waveangle stability diagram for a three-dimensional supersonic boundary layer. The growthrate curves are shown for spanwise wavelengths of $0.75,1.25$ and $1.75 \mathrm{~mm}$. The unstable frequencies range from -60 to $110 \mathrm{kHz}$. If we consider the stability for the most amplified wave with the spanwise wavelength of $1.25 \mathrm{~mm}$, the first neutral point occurs at a frequency of $\mathrm{f}=-39 \mathrm{kHz}$. The corresponding waveangle is about 92 degrees. Then with increasing frequency the growthrate increases and the growthrate and the waveangle for the stationary wave is $0.13 / \mathrm{mm}$ and 87 degrees. The growthrate peaks at a frequency of $42 \mathrm{kHz}$ and the corresponding growthrate and the waveangle are $0.20 / \mathrm{mm}$ and 80 degrees, respectively. The growthrate decreases with further increase in frequency and the second neutral point occurs at a frequency of $113 \mathrm{kHz}$ and a waveangle of 64 degrees. The most amplified waveangle for a flat plate boundary layer at a freestream Mach number of 3.0 is about 55 degrees $^{25}$. Hence, for this case the unstable waveangles are confined between 64 to 92 degrees, and the instability is mainly due to the crossflow. In the next section we will discuss how the shear layer induced by the steps alters these characteristics. Figure 9(b) shows the N-Factor results obtained from the local stability and linear PSE calculations for the traveling and stationary disturbances. The linear stability results show that the N-Factors at the transition onset location, $x=45 \mathrm{~mm}$, are about 6.5 for the traveling disturbances and about 4.8 for the stationary disturbances. The frequency and wavelength of the most amplified traveling wave are about $40 \mathrm{kHz}$ and $1.5 \mathrm{~mm}$, respectively. The wavelength of the most amplified stationary disturbances is about $1.00 \mathrm{~mm}$ to $1.25 \mathrm{~mm}$. The N-Factors obtained from linear PSE computations based on maximum $u$-velocity perturbations $u_{\max }$ are 8.0 and 6.2 for the most amplified traveling and stationary disturbances, respectively.

7 of 27

American Institute of Aeronautics and Astronautics 


\section{(b) With forward- and backward-facing steps}

Figures 11(a-d) show the stability characteristics of the boundary layers for the backward-facing step case. The step is located at $x_{s}=10 \mathrm{~mm}$ and the step height is $h_{s}=0.12 \mathrm{~mm}$. These figures depict the variation of the growthrate and waveangle as a function of the frequency for different spanwise wavelengths. The results are presented at two upstream and two downstream stations $\Delta x / h_{s}=-8,-1,2$, and 17. At the upstream station $\Delta x / h_{s}=-8$, the stability characteristics are almost the same as for the unperturbed case. At the upstream station $\Delta x / h_{s}=-1$, which is immediately upstream of the step, the growthrate decreases compared to the unperturbed case and the maximum growthrate occurs for the stationary disturbances. The growthrate of the stationary disturbances for a spanwise wavelength of $\lambda_{\mathrm{z}}=$ $1.25 \mathrm{~mm}$ with and without the steps are $0.13 / \mathrm{mm}$ and $0.16 / \mathrm{mm}$, respectively. It is also noted that the boundary layer is unstable to a broader range of high frequencies. The unstable frequency range for a spanwise wavelength of $0.75 \mathrm{~mm}$ is -180 to $85 \mathrm{kHz}$. The corresponding waveangle range is from 97 to 74 degrees. Hence the first mode in the region (1) (Fig. 10) becomes unstable to higher frequencies. The stability characteristics in the downstream stations, $\Delta x / h_{s}=2$ and 17 , are completely modified due to the separated shear layer. We note that the separated shear layer reattaches at about 10 step heights downstream of the step and the boundary layer recovers back to the unperturbed case by 84 heights downstream. The first observation is that the growthrate curves show two peaks, one in the negative frequency region and the second in the positive frequency region. The second general observation is that immediately downstream of the step, $\Delta x / h_{s}=2$, the maximum growthrates are very large on the order of $2 / \mathrm{mm}$ compared to $0.2 / \mathrm{mm}$ for the unperturbed case.

For clarity we consider the variations of the growthrate and the waveangle with the frequency for one spanwise wavelength. We select the wave with the spanwise wavelength of $1.25 \mathrm{~mm}$ for the analysis at $\Delta x / h_{s}=2$. The first neutral point occurs at a very high frequency of $-500 \mathrm{kHz}$ and at a waveangle of about 127 degrees. The growthrate then increases with the decreasing frequency and peaks at a frequency of $202 \mathrm{kHz}$ and at a waveangle of about 115 degrees. Hence these instability waves are the first mode waves (region (1) in Fig. 10) with very large growthrates produced by the shear layer. The growthrate decreases and the waveangle decreases with further decrease in frequency. The waveangle and the growthrate for the stationary disturbance are about 90 degrees and $0.48 / \mathrm{mm}$, respectively. This growthrate is about three times higher than that in the unperturbed case. The growthrate then decreases with increasing positive frequency and reaches a minimum at a frequency of $23 \mathrm{kHz}$ and at a waveangle of 86 degrees. This region is the crossflow instability region shown as region (2) in Fig. 10. With the further increase in frequency, the growthrate increases sharply and peaks at a frequency of $106 \mathrm{kHz}$ and the waveangle at this point is about 58 degrees. Beyond this point the growthrate decreases steeply and reaches the second neutral point at a frequency of $158 \mathrm{kHz}$ and at a waveangle of 37 degrees. This instability region is the second first mode instability region shown as region (3) in Fig. 10. This instability is also produced by the shear layer.

Figure 11(d) similarly shows the stability results at the station $\Delta x / h_{s}=17$ which is downstream of the reattachment point. The growthrate curves show two peaks as in the previous station. However the maximum growthrate is an order of magnitude smaller than that at the upstream station $\Delta x / h_{s}=2$ ( note the ordinate scale change in Figs. 11(c) and (d)). This is due to the weakening of the shear layer downstream. Another observation is that the maximum growthrate in the negative frequency region, or in the shear layer induced first mode instability region (1), is about two times smaller than that in the positive frequency region that consist of crossflow region (2) and shear layer induced first mode region (3) at the higher frequencies. As in the previous station, we follow the variation of the growthrate and the waveangle with the frequency for one spanwise wavelength of $1.25 \mathrm{~mm}$. The frequencies $(\mathrm{kHz})$ and the waveangles (degrees) at the four neutral points are $(-218,114),(-57,100),(-4,88)$ and $(192,42)$ and at the maximum growthrate positions are $(-139,109)$ and $(83,71)$. The maximum growthrates at the left and the right peak points are $0.18 / \mathrm{mm}$ and $.40 / \mathrm{mm}$, respectively. The growthrate for the stationary disturbance is very small $0.04 / \mathrm{mm}$ compared to $0.15 / \mathrm{mm}$ for the unperturbed case. Another intriguing observation is that the growthrate and the waveangle curves are continuous for the spanwise wavelength of $0.50 \mathrm{~mm}$. This implies that for this wavelength the modes are continuously evolving from one to another when the

8 of 27

American Institute of Aeronautics and Astronautics 
frequency changes. However with increasing wavelengths, the left modes and the right modes split and become separate. The waveangles curve for the spanwise wavelength of $1.25 \mathrm{~mm}$ shows two separate curves implying that the modes are separate and not connected to each other. This will become clear later where we consider the evolution and the integrated growth of the disturbances in the streamwise direction for a fixed spanwise wavelength.

Figures 12(a) and (b) depict the variation of the growthrates and the waveangles along the streamwise direction for stationary disturbances with different spanwise wavelengths for the backward-facing steps. In these figures we also included the results obtained for the smooth wall case. The growthrate curves can be divided into three parts. First is the part upstream of the step where the growthrates are the same as for the smooth case except immediately upstream of the step where the growthrates decrease steeply. Hence the presence of the backward-facing steps does not influence the stability characteristics of the upstream boundary layer. Second is the recirculation part immediately downstream of the step and third is the part far downstream of the step. The first observation in the third part is that the growthrates are smaller than that for the smooth case for all the wavenumbers. This shows that the effects of the step persist for a long distance downstream of the step and that the stationary crossflow instability is stabilized by the backwardfacing step. Another important observation is that when these stationary modes progress towards the step they become stable for spanwise wavenumbers larger than $0.75 \mathrm{~mm}$. The growthrate curves for the spanwise wavenumbers of 0.50 and $0.75 \mathrm{~mm}$ decrease first near the step and then continues to increase to very large values in the second part immediately downstream of the step. It is also noted that the growthrates for the spanwise wavenumber $1.25 \mathrm{~mm}$ also have large growthrates in the second part, but it does not merge with the mode downstream. This behavior was also observed for other wavenumbers larger than $0.75 \mathrm{~mm}$. These modes are induced by the shear layer and we designated them as Mode 1 in Figs. 12 (a) and (b). This is clearly seen in the waveanlge curve for the spanwise wavenumber of $1.25 \mathrm{~mm}$ shown in Fig. 12(b). The waveangle for the Mode 1 increases first and reaches a maximum value of 96 degrees before it decreases again. The waveangle for the downstream mode decreases first and reaches a minimum value of about 84 degrees before it increases. Hence crossflow vortices with shorter spanwise wavelengths below $0.75 \mathrm{~mm}$ will have substantial growth in the recirculation part before they progress downstream. The crossflow vortices with longer wavelengths will decay first in the recirculation part before they grow further downstream.

Figures 13(a,b) show the integrated growth, N-Factors, of the instability waves for different spanwise wavelengths of $0.50,0.75,1.25$ and $1.50 \mathrm{~mm}$. Figure 13(a) shows the integrated growth in parts 1,2 and 3 separately. In Figure 13(b), we present the total growth by summing the growth in regions 1 to 3 . This is the usual practice employed in calculating the N-Factors behind steps ${ }^{13-17}$. The assumption in this approach is that the modifications to the amplitude of the crossflow vortices across the step, i.e. the receptivity due to the step, are negligible. We will address this question from our direct numerical simulation (DNS) in a later section. In Figure 13(b), solid lines depict the N-Factor variations with the step and the dotted lines depict the results for the smooth case. The growth of the instability waves with the shorter wavelengths of less than $0.75 \mathrm{~mm}$ reach large values in the region 2 as expected from the growthrate curves shown in Fig. 12(a). This makes the total N-Factor for the shorter wavelengths 0.50 and $0.75 \mathrm{~mm}$ larger than that for the smooth case. The maximum N-Factor for the wavelength of $0.50 \mathrm{~mm}$ increases from 2.2 to 3.5 at $x=15 \mathrm{~mm}$. The absolute numbers and the increments may be small in this case, but it suggests that with increasing step heights the N-Factors may reach large values near the steps and may cause early transition. The integrated growth of Mode 1 for the wavelengths $1.00,1.25$ and 1.50 $\mathrm{mm}$ are small on the order of 0.4 , hence they may not be important in the transition scenario. The figure also shows that the disturbances with wavelengths of 1.25 and $1.50 \mathrm{~mm}$ start to grow a few step heights downstream of the step. These two cumulative effects, the stabilization of the boundary layer and the delay in the amplification, produce smaller N-Factors compared to the smooth case beyond $x=20 \mathrm{~mm}$. The N-Factors at the smooth surface transition onset point $x=45 \mathrm{~mm}$ are 4.0 and 4.8 with and without the step for the most amplified spanwise wavenumber of $1.25 \mathrm{~mm}$. Hence the amplitudes will be reduced by about 2.2 times near $x=45 \mathrm{~mm}$.

9 of 27 
Figures 14 and 15 show the variation of the growthrates and the integrated growthrates, N-Factors, for the traveling disturbances for the most amplified frequencies and spanwise wavelengths for the backwardfacing step case. We plotted the results for frequencies and wavelengths that give the largest N-Factors along the $x$ direction. Figure 14 shows the variation of the growthrates and the waveangles along the streamwise direction. The results are shown for one medium frequency shear layer first mode $(-63 \mathrm{kHz}$, $0.50 \mathrm{~mm})$, one traveling crossflow mode $(40 \mathrm{kHz}, 1.25 \mathrm{~mm})$ and one medium frequency second first mode $(79 \mathrm{kHz}, 0.75 \mathrm{~mm})$. It is noted that the mode $(40 \mathrm{kHz}, 1.25 \mathrm{~mm})$ gives the largest N-Factor at the transition onset point in the smooth case. As was discussed previously, the growthrate curves can be divided into three parts. The growthrates are the same as for the smooth case in the part upstream of the step except immediately upstream where the growthrates decrease steeply in a narrow region. Hence the backward-facing steps do not influence the stability characteristics of the traveling waves upstream as was observed for the stationary waves. In the third part, the growthrates are smaller than that for the smooth case similar to the stationary disturbances. This shows that traveling disturbances are also stabilized by the backward-facing step. In part 2, we observe the growth of the first mode unstable waves that were induced by the shear. The growthrate for the medium frequency mode $(-63 \mathrm{kHz}, 0.5 \mathrm{~mm})$ is about 8 times higher than the maximum growthrate for the smooth case. However, the growthrates decay steeply and become small a short distance downstream. The waveangle for this wave is in the range of 100 degrees. The second medium range first mode $(79 \mathrm{kHz}, 0.75 \mathrm{~mm})$ has larger growthrates than the smooth case for a longer distance up to $x=15 \mathrm{~mm}$, and hence will produce higher N-Factors compared to the smooth case. The most amplified mode in the smooth case $(40 \mathrm{kHz}, 1.25 \mathrm{~mm})$ first decayed immediately downstream of the step before it grows further downstream. Similar to the stationary disturbances case, Figures 15(a) and (b) depict the integrated growthrates for different modes. Figure 15(a) shows the growth in the three parts separately and Figure 15(b) shows the total growth obtained by summing the N-Factors in the upstream and downstream regions. The N-Factors at $x=20 \mathrm{~mm}$ are 5.0 and 4.5 with and without the step. Beyond this point, the traveling disturbances are stabilized by the backward-facing step. The N-Factors for the traveling wave with frequency of $40 \mathrm{kHz}$ and a spanwise wavelength of $1.25 \mathrm{~mm}$ are 5.85 and 6.6 at $x=45 \mathrm{~mm}$ with and without steps, respectively. Hence the amplitudes will be about two times smaller with the step compared to that without the step. The maximum N-Factor obtained for the medium frequency shear layer instability $(-63 \mathrm{kHz}, 0.5 \mathrm{~mm})$ is about 1.8 , which is smaller than the traveling crossflow instability for this case. With increasing step heights these high frequency first modes may play more of a role in the transition behind the steps.

Similar to the backward-facing step, Figures $16(\mathrm{a}-\mathrm{d})$ show the stability characteristics of the boundary layer with a forward-facing step. The step is again located at $x_{s}=10 \mathrm{~mm}$ and the step height is also $h_{s}=$ $0.12 \mathrm{~mm}$. The results are presented at three upstream stations and one downstream station $\Delta x / h_{s}=-17,-8$, -1 , and 2 . We note that the flow separates at about 9 step heights upstream of the step and the boundary layer begins to deviate from the unperturbed case at about 25 heights upstream. At the upstream station $\Delta x / h_{s}=-17$, the stability characteristics are similar to the unperturbed case. However, the growthrates are slightly larger with the step compared to the smooth case. The maximum growthrates for a spanwise wavelength of $1.25 \mathrm{~mm}$ are $0.32 / \mathrm{mm}$ and $0.24 / \mathrm{mm}$ with and without the step, respectively. At the downstream station $\Delta x / h_{s}=1$, which is immediately downstream of the step, the growthrate decreases compared to the unperturbed case and the maximum growthrate occurs close to the stationary disturbances. The growthrate of the stationary disturbances for a spanwise wavelenghth of $\lambda_{\mathrm{z}}=1.25 \mathrm{~mm}$ with and without the steps are $0.10 / \mathrm{mm}$ and $0.16 / \mathrm{mm}$, respectively. The stability characteristics in the separated region, $\Delta x / h_{s}=-8$ and -1 , are similar to the characteristics for the backward-facing step. The growthrate curves show two peaks: one in the negative frequency region and the second in the positive frequency region. The instability in the negative frequency region is the first mode instability enhanced by the shear layer. The instability in the positive frequency side is due to the crossflow and the first mode. The growthrates in this case are about two times smaller than that for the backward-facing step case. The maximum growthrate for a spanwise wavelength of $1.25 \mathrm{~mm}$ is about $1.1 / \mathrm{mm}$ compared to $1.9 / \mathrm{mm}$ for

10 of 27

American Institute of Aeronautics and Astronautics 
the backward-facing step. This is due to the weaker shear layer in the forward-facing step compared to that for the backward-facing step.

Figures 17(a) and (b) depict the variations of the growthrates and the N-Factors along the streamwise direction for different spanwise wavelengths. As we discussed previously, the growthrate curve can be divided into three parts: upstream, downstream and close to the step. In the upstream and downstream parts, the growthrates are the same as that for the smooth case. In the upstream region close to the step, the growthrate for the shorter wavelength, $0.50 \mathrm{~mm}$, grows steeply starting at $x=8 \mathrm{~mm}$ and reaches a maximum value immediately upstream of the step. However, the growthrate for this wave decreases below the smooth case downstream of the step. We also included the variation of the waveangle for this wave in the figure. It is seen that the waveangle increases from about 85 degrees for the smooth case to about 91 degrees with the step. This mode shifts towards the crossflow direction. The growthrates for the higher spanwise wavelengths, 0.75 and $1.25 \mathrm{~mm}$, decrease below the smooth case in the region close to the step. Another mode with large growthrates exists very close to the step, but the upstream mode and this new mode remain separate. We observed the appearance of an additional mode in the backwardfacing step also, and we need to investigate the consequence of this further. The N-Factor curves show that the integrated growth with the step is smaller than for the smooth case for the longer wavelengths. This is due to the stabilization of the growth of these waves near the step. The N-Factor for the wave with the shorter wavelength of $0.50 \mathrm{~mm}$ increases upstream of the step due to the high growth upstream of the step. However, the increase in the N-Factor is small for this step height of $0.12 \mathrm{~mm}$ (on the order of 0.4).

Similarly, Figures 18(a,b) show the variation of the growthrates and the N-Factors for the most amplified traveling disturbances for the forward-facing step. Upstream of the step, the high frequency wave $(79 \mathrm{kHz}, 0.50 \mathrm{~mm})$ has a larger growthrate than that for the smooth case. However, this mode becomes stable downstream of the step. For the most amplified wave in the smooth case $(47 \mathrm{kHz}, 1.00$ $\mathrm{mm}$ ), the step case has larger growthrates than that in the smooth case in some region and smaller growthrates downstream. Hence they do not produce a net increase in the N-Factors across the step. The most amplified waves induced by the shear $(-95 \mathrm{kHz}, 1.50 \mathrm{~mm})$ have very large growthrates in a narrow part immediately upstream of the step but the integrated growth is small on the order of 1.2 in this case. Hence the forward-facing step at this height $\left(h_{s}=0.12 \mathrm{~mm}\right)$ does not make any noticeable change in the integrated growth.

\section{Evolution of stationary crossflow vortices}

\section{a. Without steps}

We first performed direct numerical simulations for the swept wing with roughness elements placed near the neutral point but without the steps. The parameters $x_{r}, \Delta x_{r}, h_{r}$ and $\sigma_{r}$ are given in Table 3 . The boundary-layer thickness near $x=1.0 \mathrm{~mm}$ is about $0.075 \mathrm{~mm}$. Simulations were performed for the most amplified wave with the spanwise wavelength $\lambda_{z}=1.25 \mathrm{~mm}$ and for roughness heights, $h_{r}$, of $1 \mu \mathrm{m}$. The roughness Reynolds number, $\operatorname{Re}_{k, u}=|u|_{\mathrm{k}} h / v_{k}$, for these parameters is about 0.6. Detailed results about the evolution and the receptivity coefficients of the crossflow vortices generated by the roughness were presented in a previous paper ${ }^{5}$.

Table 3. Location and the height of the roughness on the wing

\begin{tabular}{cccccc}
\hline$x_{r}(\mathrm{~mm})$ & $h_{r}(\mathrm{~mm})$ & $\sigma_{r}$ & $\Delta x_{r}(\mathrm{~mm})$ & $h_{r} / \delta$ & $R e_{k, u}$ \\
\hline 1 & 0.001 & 3 & 0.25 & $1 / 75$ & 0.6
\end{tabular}

Figure 19 depicts the contours of the $u$ velocity at half the boundary-layer height in the plan view $(x, z)$ plane for the case $x_{r}=1.0 \mathrm{~mm}$ and $h_{r}=0.001 \mathrm{~mm}$. These striations are the footprints of the stationary crossflow vortices that originate from the roughness elements. Figures 20 (a, b) show the perturbations of the maximum $u$ velocity generated by the roughness elements in linear and log scales, respectively. We

11 of 27 
$44^{\text {th }}$ AIAA Fluid Dynamics Conference, June 16-20, 2014, Atlanta, Georgia

also included the amplitude of the perturbations computed using linear PSE. The agreement is very good in the linear region but the computations from the linear theory deviate when the maximum $u$-velocity perturbations reach about 0.18 . This is equivalent to 0.28 when it is nondimensionalized by the boundary-layer edge velocity.

\section{b. With forward- and backward-facing steps}

Numerical simulations were performed to investigate the effects of forward- and backward- facing steps on the crossflow vortices. Simulations were performed with forward- and backward-facing steps placed at different stations $\left(x_{s}=5,10\right.$ and $15 \mathrm{~mm}$ ) with increasing heights. The results are first presented for the forward- and backward-facing steps located at $x_{s}=10 \mathrm{~mm}$ with increasing step heights of $h_{s}=0.06$, 0.12 , and $0.18 \mathrm{~mm}$.

Figures 21(a,b) show the evolution of the crossflow vortices over the forward- and backward-facing step in the $(x, z)$ plane at half the boundary layer height, respectively. The steps are located at $x_{s}=10 \mathrm{~mm}$ and the step height is $h_{s}=0.12 \mathrm{~mm}$. It can be seen that the vortices are only locally modulated by the steps and in a short distance downstream the crossflow vortices follow a trajectory similar to that observed in Fig. 19 for the case without the step. Figures 22 (a-c) show the contours of the perturbation $u$ velocity in the cross sectional $(x, y)$ plane at $z=0$. Figure 22(a) shows the results without the steps and Figs. 22(b) and (c) show the results for the forward- and backward-facing steps, respectively. It is seen that the crossflow vortices evolve across the steps without much modulation. The amplitude of the vortices increases by a small amount across the forward-facing step and decreases by a small amount across the backward-facing step.

Figures 23 (a-f) show the quantitative comparison of the growth of the maximum crossflow $u$-velocity disturbances along the wing with and without the forward- and backward-facing steps. Figures 23 (a, b, c) depict the results for the forward-facing steps located at $x_{s}=10 \mathrm{~mm}$ with increasing step heights $h_{s}=0.06$, 0.12 , and $0.18 \mathrm{~mm}$ respectively, while Figs. $11(\mathrm{~d}, \mathrm{e}, \mathrm{f})$ show the results for the backward-facing steps. It is seen that the forward-facing steps do not modify the amplitude. The backward-facing steps reduce the amplitude of the stationary crossflow vortices by a large amount. The decrease in the amplitude for the backward-facing step increases with the height. The amplitude decreases by factors of $3 / 4,2 / 3$ and $1 / 2$ at the streamwise station $x=30 \mathrm{~mm}$ for the step heights $h_{s}=0.06,0.12$, and $0.18 \mathrm{~mm}$, respectively. Figure 24 shows the variation of the maximum $u$-velocity disturbance amplitude along the streamwise direction for the forward- and backward-facing steps. An important observation is that the amplitudes of the fluctuations in the immediate vicinity of the steps increase upstream for the forward-facing step and decrease downstream for the backward-facing step. For the backward-facing step, the amplitude curves remain parallel to the smooth case but at smaller amplitudes. For the forward-facing step, the amplitudes grow slower than that for the smooth case and merge with the amplitude for the smooth case.

Figures $25(\mathrm{a}, \mathrm{b})$ depict the $u$-velocity perturbations generated by the roughness and a backward-facing step for shorter wavelengths, $\lambda_{z}=0.50$ and $0.75 \mathrm{~mm}$. The step is located at $x_{s}=10 \mathrm{~mm}$ and the step heights are $h_{s}=0.12$ and $0.18 \mathrm{~mm}$. Interestingly as we predicted by the linear theory (Fig. 12(a)), the disturbances grow substantially immediately downstream of the step consistent with linear theory. It is also seen that the amplitude growth downstream of the step increases with the height. The amplitude grows from 0.01 to 0.044 from $x=10$ to 13 for the spanwise wavelength of $0.50 \mathrm{~mm}$ and the height of 0.12 $\mathrm{mm}$ and it grows from 0.01 to 0.11 for the step height of $0.18 \mathrm{~mm}$. Similarly for the spanwise wavelength of $0.75 \mathrm{~mm}$, the amplitude grows from 0.027 to 0.07 for the step height of $0.12 \mathrm{~mm}$ and it grows to 0.15 for the step height of $0.18 \mathrm{~mm}$. After that the disturbance amplitudes decrease before they grow again downstream. This behavior does not agree with the linear stability predictions which indicate the disturbance amplitude should continuously grow downstream of the step but at a slower rate. We need to explore this in the future. In Figure 26 we present the maximum amplitude variation obtained with a backward-facing step of heights $h_{s}=0.12$ and $0.18 \mathrm{~mm}$ for different spanwise wavelengths $\lambda_{z}=1.25$, $1.00,0.75$ and $0.50 \mathrm{~mm}$. It is interesting to observe that the amplitudes for the longer wavelengths $\lambda_{z}=$ 1.25 and $1.00 \mathrm{~mm}$ decay downstream of the step while the shorter wavelengths $\lambda_{z}=0.50$ and $0.75 \mathrm{~mm}$ increase steeply downstream of the step. This local increase agrees with the linear stability prediction.

12 of 27

American Institute of Aeronautics and Astronautics 
Additionally, we did not observe any impulsive change in the amplitude at the step. The changes take place within the recirculation zone. Hence the receptivity defined as the change at the step (receptivity site) does not occur for the cases we explored.

\section{Conclusions}

The effects of forward- and backward-facing steps on receptivity and stability of three-dimensional supersonic boundary layers over a swept wing with a blunt leading edge were numerically investigated for a freestream Mach number of 3. Computations were performed for several step locations along the streamwise direction and for several step heights. The heights varied from one third to one boundary layer thickness. The forward- and backward-facing steps created small separation zones upstream and downstream of the steps, respectively. The separation lengths for the forward-facing step varied between 5 to 10 step heights where the separation length increased with increasing step height. The boundary layer deviated from the unperturbed case about 25-60 step heights upstream and recoverd back to the unperturbed case by $80-125$ step heights downstream. The separation lengths for the backward-facing steps were about 10 step heights and did not appreciably change with the location or the height. The boundary layer deviates from the unperturbed case from 10-25 step heights upstream and recovered by about 80-125 steps downstream. The boundary layer showed strong inflectional profiles in the vicinity of the step. The backflow velocity in the separation zones for the forward-facing steps were about 3 times smaller than that for the backward-facing steps.

Linear stability computations revealed strong shear-layer induced instabilities in the separated region. Compared to the classical crossflow stability characteristics, the stability results showed two peaks with very large growthrates and a broad unstable frequency range. One is in the quadrant between the negative crossflow direction and the inviscid streamline (regions 2 and 3 in Fig. 10) and the second is in the quadrant between the crossflow and the inviscid streamline (region 1 or 4 in Fig. 10). These instabilities are induced by the strong shear layer that exists in the separated region.

The variation of the growthrates along the streamwise direction showed that the growthrates were not affected by the backward-facing steps upstream. Further downstream of the steps, the growthrates for the most amplified disturbances, for both stationary and traveling, were stabilized by the steps. Immediately downstream of the backward-facing step, the growthrates of the most amplified waves were damped. Stationary disturbances with shorter spanwise wavelengths had larger growthrates in the vicinity immediately downstream of the step. This may have implications in the transition induced by the steps. The waves with the larger spanwise wavelengths also had large growthrates immediately downstream of the step, but they did not merge with the mode further downstream. Hence they did not contribute to the integrated growth, N-Factors, downstream. The N-Factors obtained for the most amplified waves induced by the shear were small (on the order of 1.8 or less) for the cases considered in the computations.

The simulation of the stationary crossflow vortices with the steps showed that the forward-facing steps did not appreciably modify the amplitudes of the crossflow vortices. The backward-facing steps decreased the amplitudes of the crossflow vortices with longer wavelengths by about fifty percent near the transition onset point for the unperturbed case. The amplitudes of the waves with the shorter wavelengths increases substantially immediately downstream of the backward-facing step before they decay again. These behaviors qualitatively agree with the linear stability calculations. However, according to linear stability the shorter wavelength vortices should have continuously grown, but at a smaller rate, contrary to the simulation results which show a decay. This difference needs to be investigated further. Hence if the step heights are large enough the shorter wavelength stationary crossflow vortices can potentially cause abrupt transition near the step.

\section{References}

1. Holmes, B. J., "Progress in Natural Laminar Flow Research,” AIAA Paper 84-222, 1984.

13 of 27

American Institute of Aeronautics and Astronautics 
2. Drake, A., Bender, A. M., Korntheuer, A. J., Westphal, R. V., McKeon, B. J. and Gerashchenko, S. "Step Excrescence Effects for Manufacturing Tolerances on Laminar Flow Wings," AIAA Paper 2010-375, 2010.

3. Bippes, H., "Basic Experiments on Transition in Three-Dimensional Boundary Layers Dominated by Crossflow Instability," Progress in Aerospace Sciences, Vol. 35, pp. 363-412, 1999.

4. Saric, W. S., Reed, H. L., and White, E. B., "Stability and Transition of Three-Dimensional Boundary Layers," Annual Review of Fluid Mechanics, Vol. 35, pp. 413-440, 2003.

5. Balakumar, P., and King, R. A., "Receptivity to Roughness, Acoustic, and Vortical Disturbances in Supersonic Boundary Layers Over Swept Wings,” AIAA Paper 2011-3880, 2011.

6. Balakumar, P., and King, R. A., "Receptivity and Transition of Supersonic Boundary Layers Over Swept Wings," AIAA Journal, Vol 50, Number 7, July 2012, pp. 1476-1489.

7. Fage, A. "The Smallest Size of Spanwise Surface Corrugation which Affects Boundary Layer Transition on an Aerofoil," R\&M No 2120, A.R.C Technical Report, 1943.

8. Carmichael, B. H. "Surface Waviness Criteria for Swept and Unswept Laminar Suction Wing," Norair Report No NOR-59-438 (BLC-123).

9. Holmes, B. J., Obara, C. J., Martin, G. L., and Domack, C. S. "Manufacturing Tolerances for Natural Laminar Flow Airframe Surfaces," SAE Paper 85-0863, General Aviation Meeting and Exposition, Wichita, Kansas, 1985.

10. Klebanoff, P. S., Cleveland, W. G., and Tidstrom, K. D., "On the Evolution of a Turbulent Boundary layer Induced by a Three-dimensional Roughness Element," J. Fluid Mech. (1992), vol. 237, pp. 101-187.

11. Nayfeh, A. H., Ragab, S. A., and Al-Maaitah, A. A. "Effects of Bulges on the Stability of Boundary Layers," Physics of Fluids, No 31(4), 1988.

12. Massad, J. A., and Iyer, V. "Transition Prediction and Control in Subsonic Flow over a Hump," Physics of Fluids, Vol 6, pp. 313-327, 1994.

13. Crouch, J. D., Kosorygin, V. S., and Ng, L. L., "Modeling the Effects of Steps on Boundary Layer Transition," Sixth IUTAM Symposium on "Laminar-Turbulent Transition," Bangalore, India, Springer, R. Govindarajan Ed., 2006.

14. Arnal, D., Perraud, J., and Seraudie, A. "Attachment Line and Surface Imperfection Problems," RTOAVT/VKI Lecture Series "Advances in Laminar-Turbulent Transition Modelling”, 2008.

15. Perraud, J., Seraudie, A "Effects of Steps and Gaps on 2D and 3D Transition," ECCOMAS 2000, Barcelona, 11-14 September 2000.

16. Perraud, J., Seraudie, A., Reneaux, J., Arnal, D., and Tran, D. "Effect of 2D and 3D Imperfections on Laminar-Turbulent Transition," CEAS Katnet Conference on Key Aerodynamic Technologies, Bremen, Germany, 2005.

17. Edelman, C. A., and Rist, U. "Impact of Forward-Facing Steps on Laminar-Turbulent Transition in Transonic Flows without Pressure Gradient," AIAA Paper 2013-0080, 2013.

18. Nenni, J. P., and Gluyas, G. L. "Aerodynamic Design and Analysis of an LFC surface," Astronautics and Aeronautics, Vol 4, pp. 52-57, 1966.

19. Cebeci, T., and Ergan, D. A. "Prediction of Transition due to Isolated Roughness," AIAA Journal, Vol 27, pp. 870-875, 1989.

20. Wie, Y., and Malik, M. R., "Effect of Surface Hardness on Boundary Layer Transition in TwoDimensional flow," Computers \& Fluids, Vol 27(2), 157-181, 1998.

21. Worner, A., Rist, U., and Wagner, S. "Influence of Humps and Steps on the Stability Characteristics of a 2D Laminar Boundary Layer," AIAA Paper 2002-0139, 2002. 
22. Eppink, J.L., "The Interaction of a Backward Facing Step and Crossflow Instabilities in Swept Boundary Layer Transition," PhD Dissertation, Mechanical Engineering Department, Tufts University, Medford, MA, 2014.

23. Archambaud, J. P., Louis, F., Séraudie, A., Arnal, D. and Carrier, G., "Natural Transition in Supersonic Flows: Flat Plate, Swept Cylinder, Swept Wing,” AIAA Paper 2004-2245, 2004.

24. Chen, Z., Yi ShiHe, He Lin, Tian LiFeng and Zhu YangZhu, "An experimental study on fine structures of supersonic laminar/turbulent flow over a backward-facing step based on NPLS", Fluid Mechanics, Chinese Science Bulletin, February 2012, Vol. 57, N0.6: 584-590.

25. Mack, L., “Boundary Layer Stability Theory,” JPL Report No. 900-277 Rev. A, Pasadena, Calif, Nov. 1969. 
(a) Swept wing

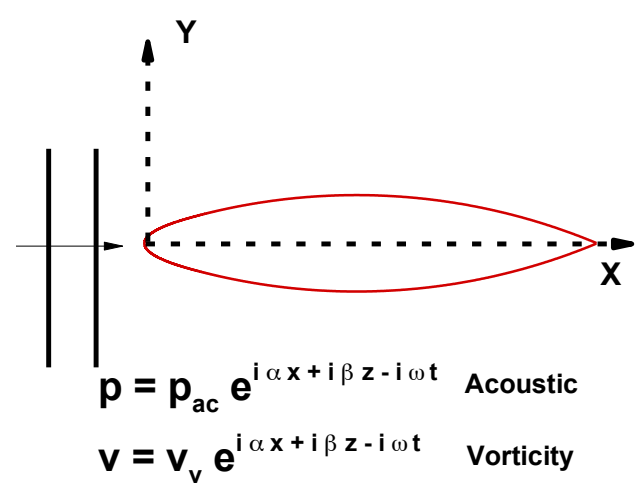

(c) Forward facing step

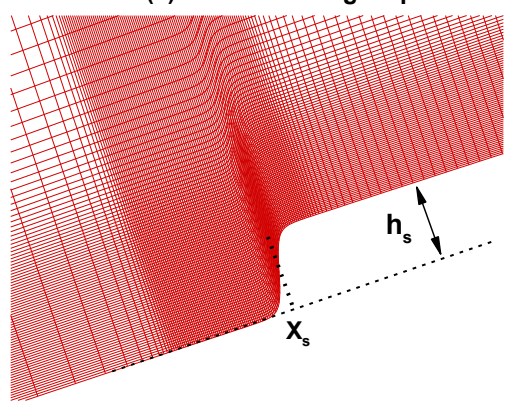

(b) Swept wing with roughness and step

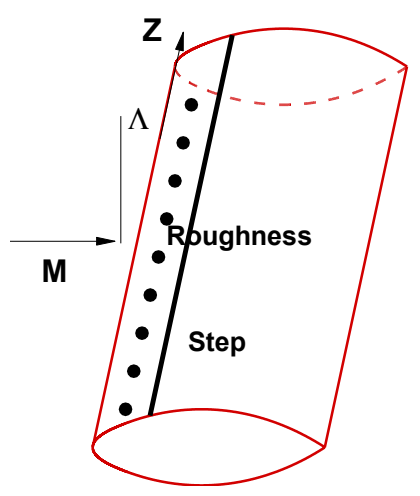

(d) Backward facing step

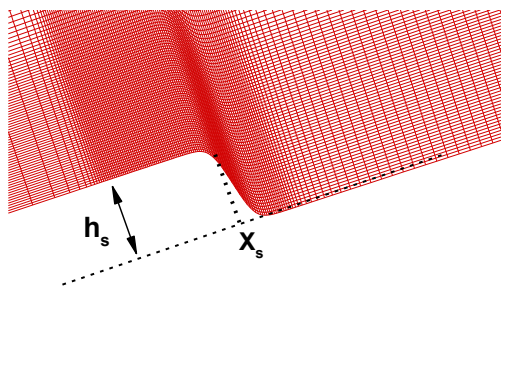

Figure 1. Supersonic flow over an infinite biconvex blunt wing with a step.

(a)

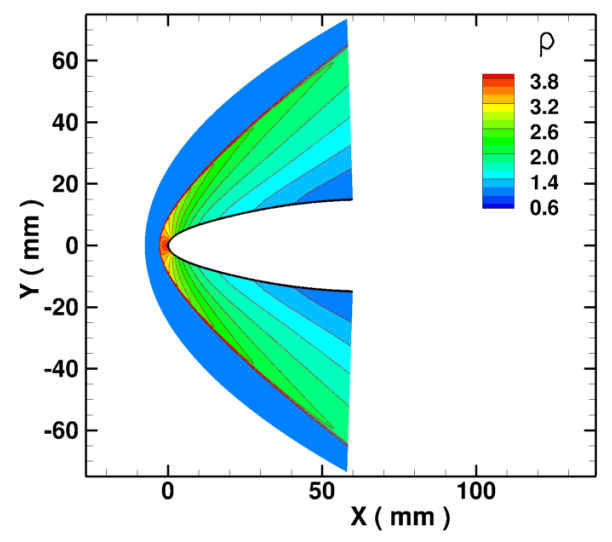

(b)

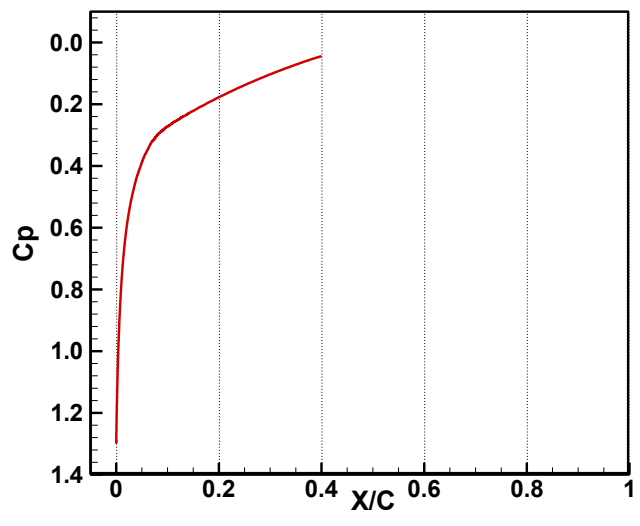

Figure 2. (a) Contours of density and (b) the pressure coefficient for flow over an infinite swept wing with a blunt leading edge at $M=3.0$. 

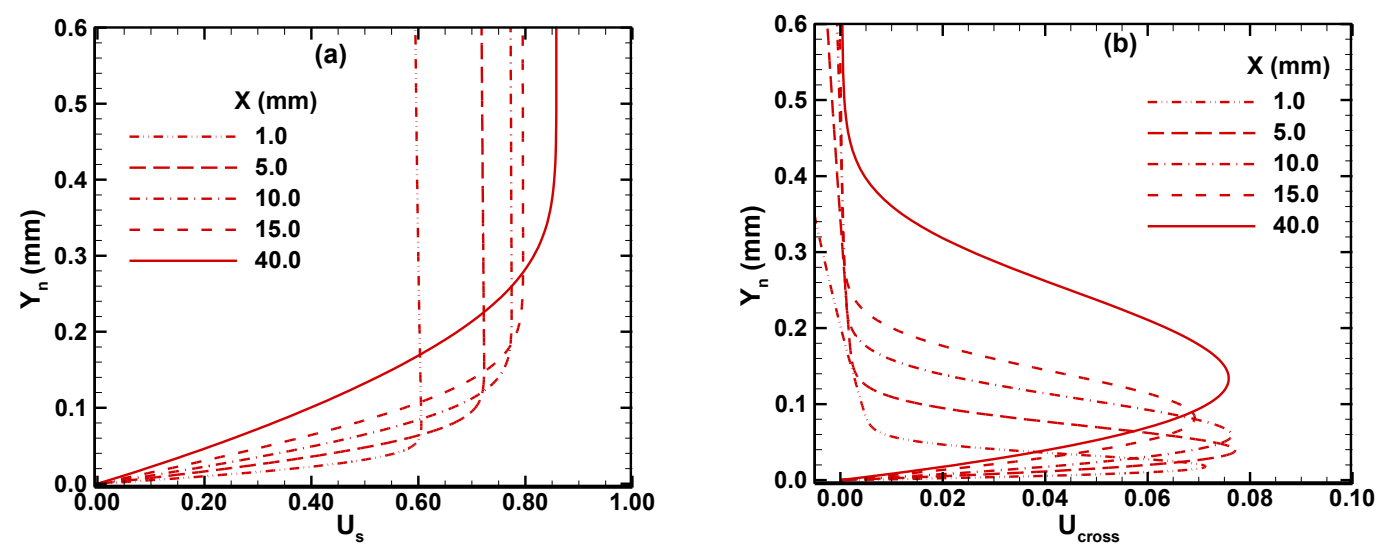

Figure 3. Computed boundary-layer velocity profiles without the step at different stations: (a) the velocity profiles in the direction of streamlines and (b) the crossflow velocity profiles.

(a)

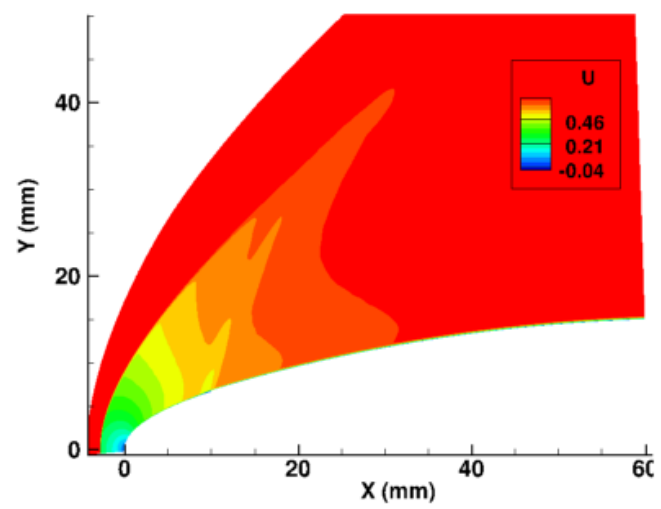

(b)

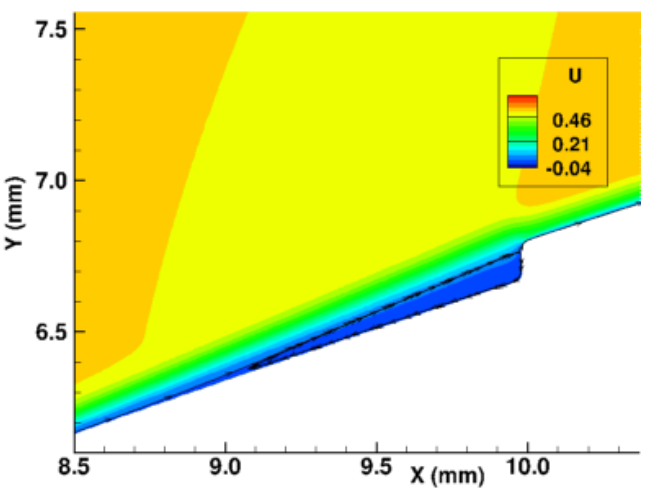

Figure 4. Contours of u velocity computed with a forward-facing step located at $x_{s}=10 \mathrm{~mm}$ and $h_{s}=0.12$ $\mathrm{mm}$ : (a) the contours over the wing and (b) the contours and the streamlines across the step.

(a)

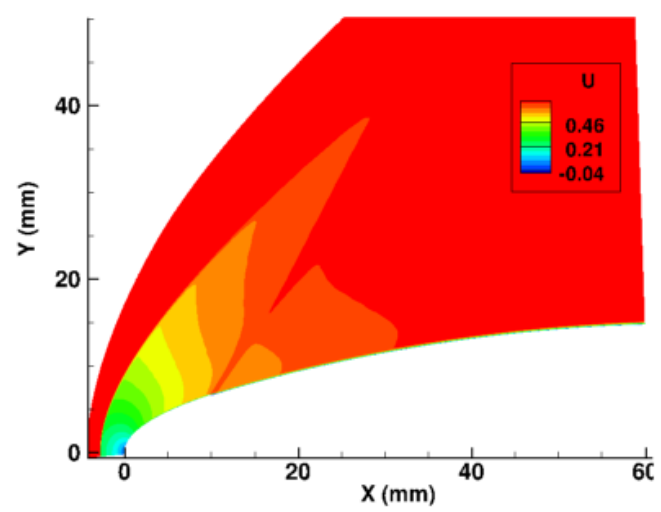

(b)

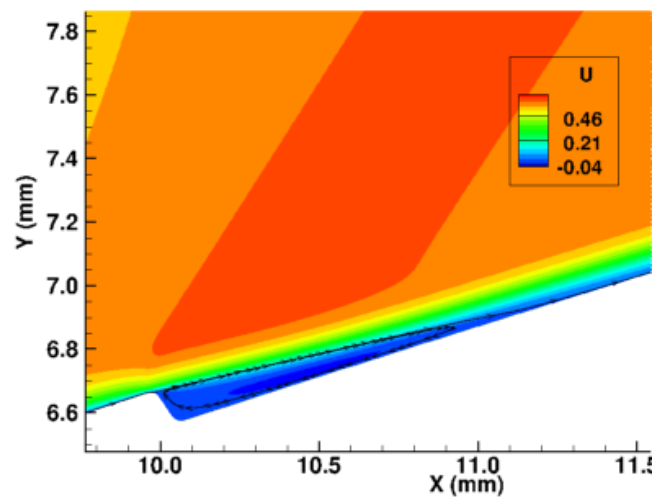

Figure 5. Contours of u velocity computed with a backward-facing step located at $x_{s}=10 \mathrm{~mm}$ and $h_{s}=0.12$ mm: (a) the contours over the wing and (b) the contours and the streamlines across the step. 


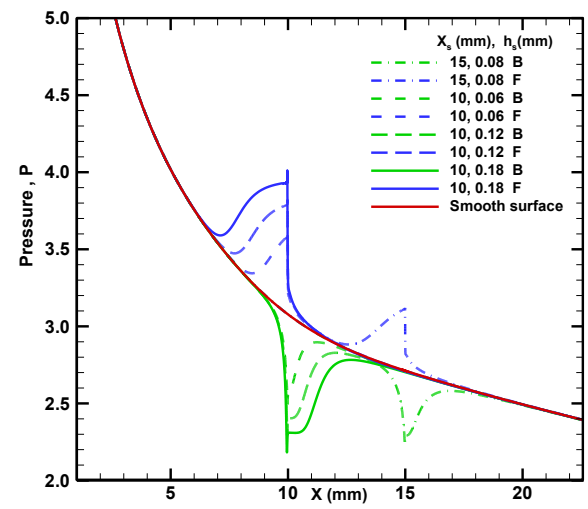

Figure 6. Surface pressure distributions for the flow over forward (F)- and backward (B)- facing steps.
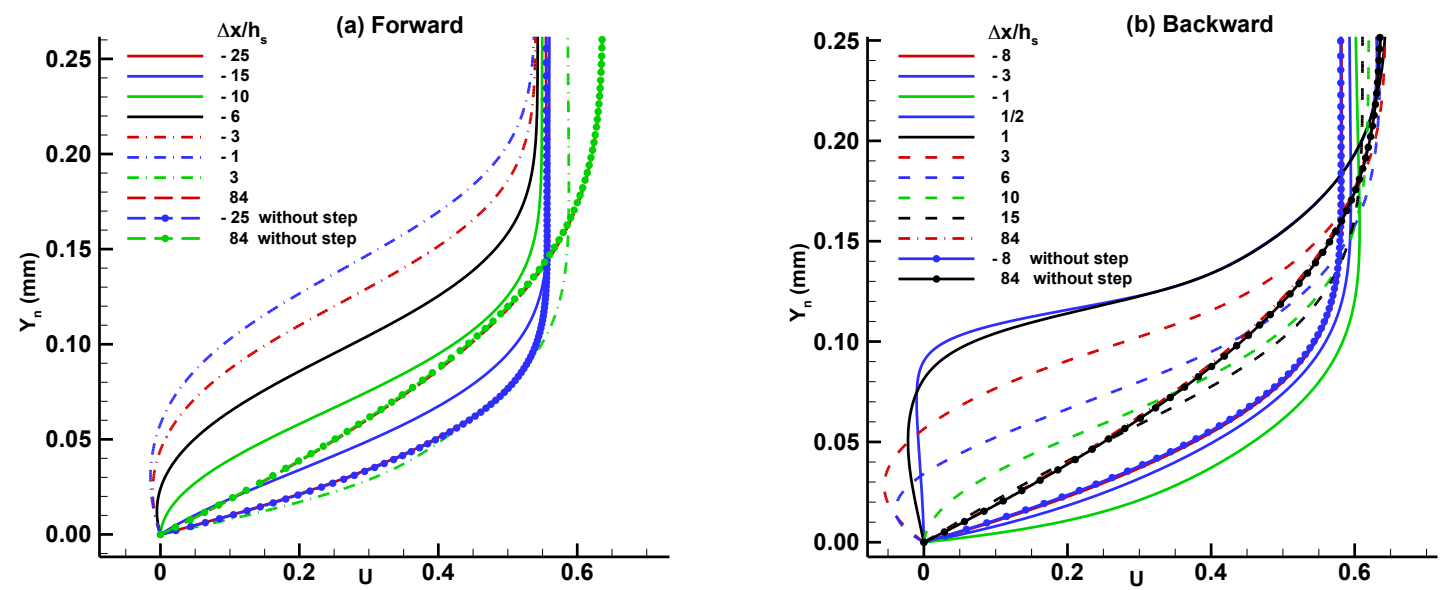

Figure 7. Computed boundary-layer velocity profiles at different stations induced by (a) forward- and (b) backward-facing steps $\left(x_{s}=10 \mathrm{~mm}, h_{s}=0.12 \mathrm{~mm}\right)$.
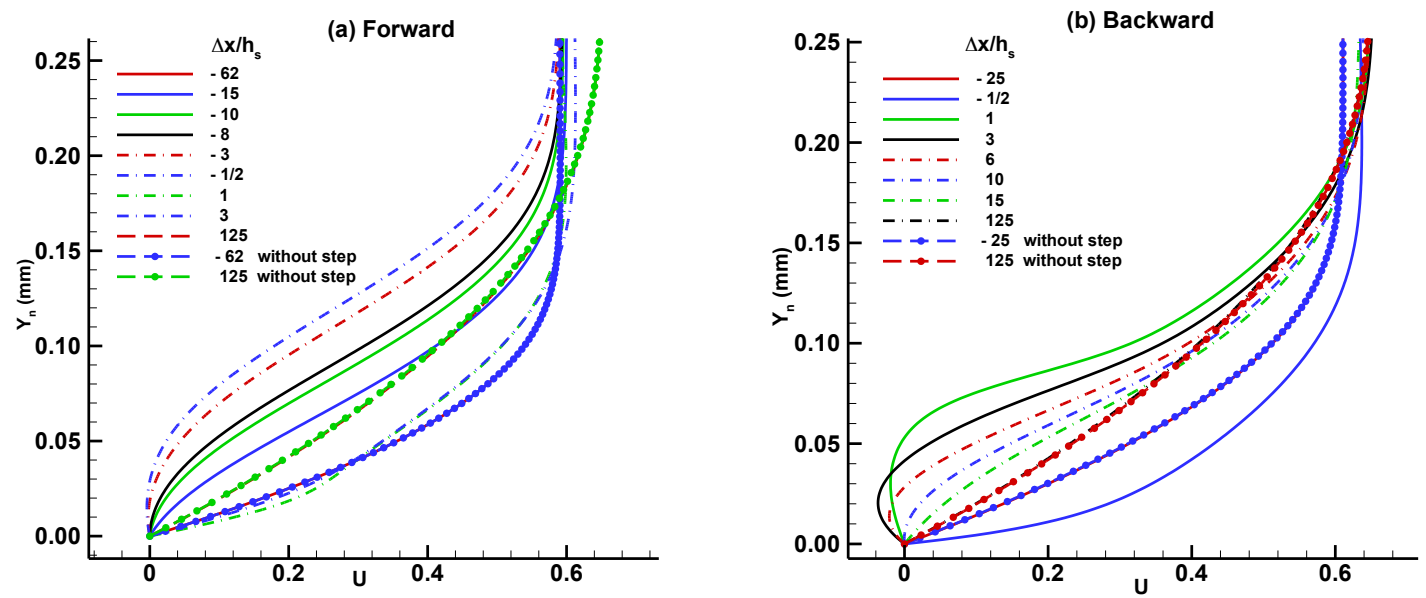

Figure 8. Computed boundary-layer velocity profiles at different stations induced by (a) forward- and (b) backward-facing steps ( $\left.x_{s}=15 \mathrm{~mm}, h_{s}=0.08 \mathrm{~mm}\right)$. 

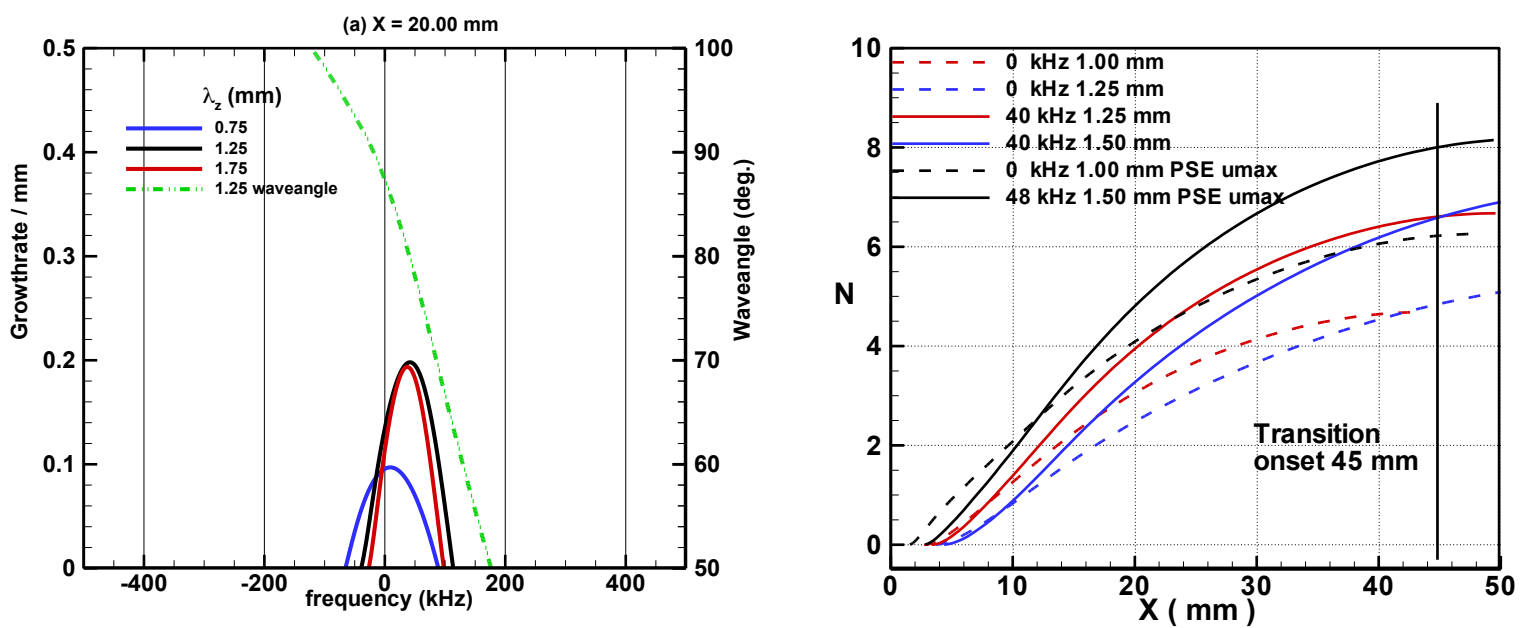

Figure 9. (a) Growthrate and (b) 1Gctors for stationary and traveling disturbances without the step.

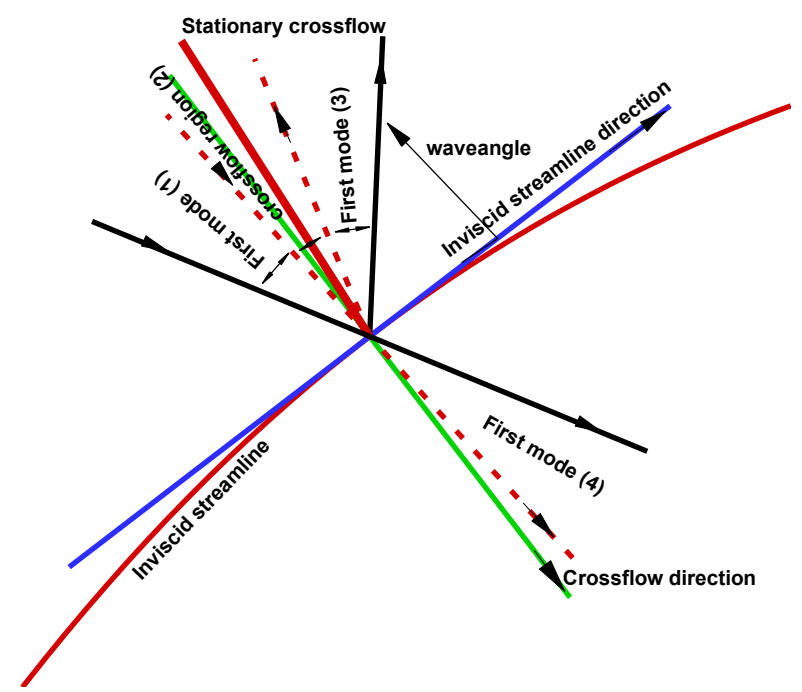

Figure 10. Schematic diagram showing the waveangle distribution for the first mode and crossflow instabilities.
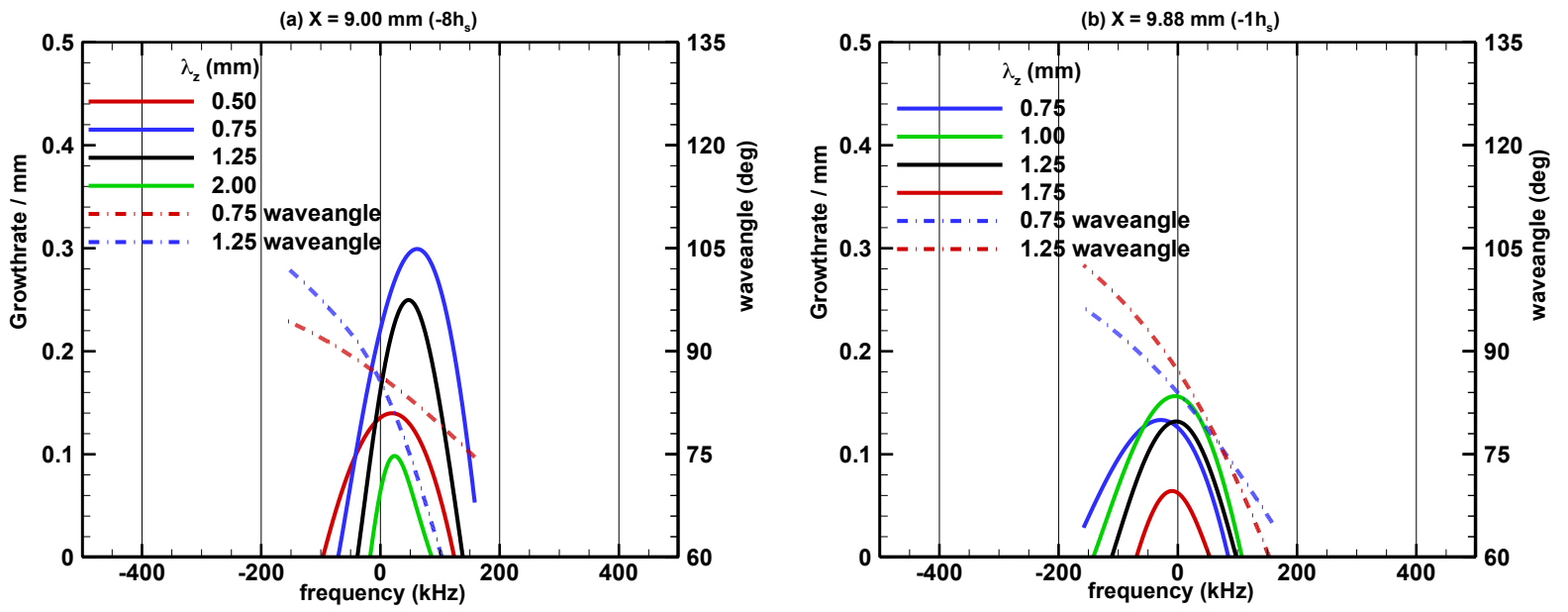

19 of 27

American Institute of Aeronautics and Astronautics 

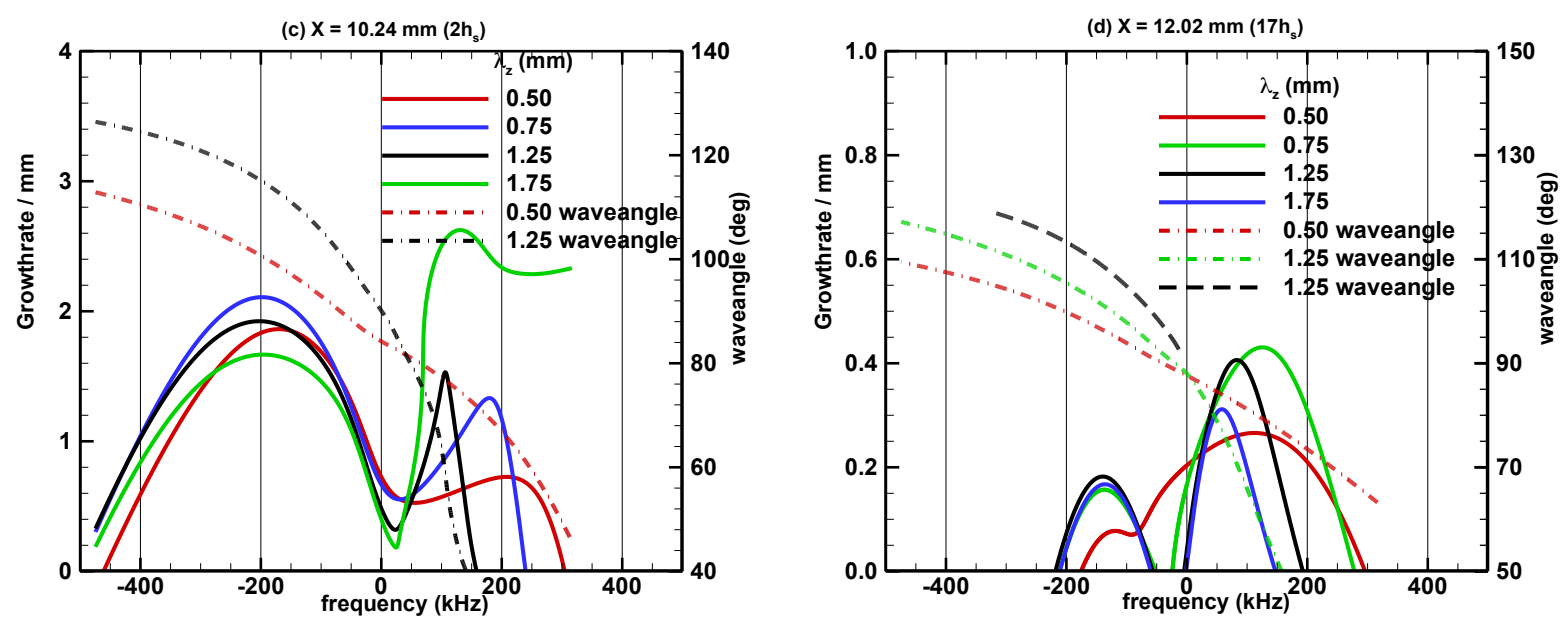

Figure 11. Variation of growthrates with the frequency for different spanwise wavelengths at upstream and downstream locations of a backward-facing step $\left(x_{s}=10 \mathrm{~mm}, h_{s}=0.12 \mathrm{~mm}\right)$.

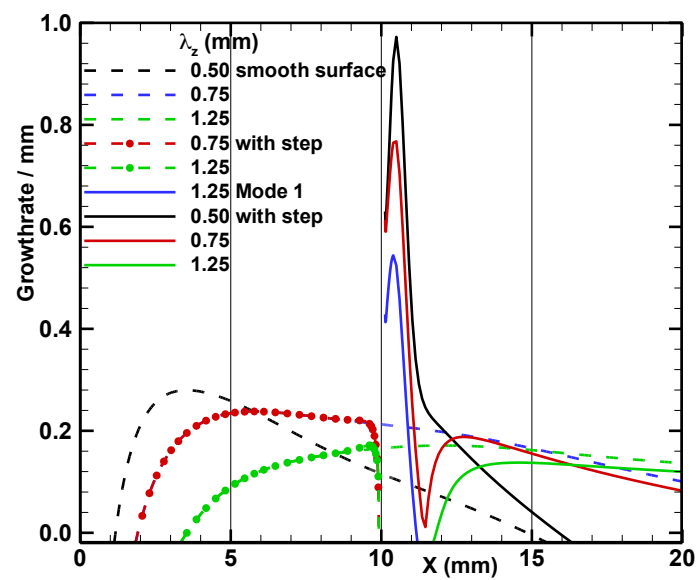

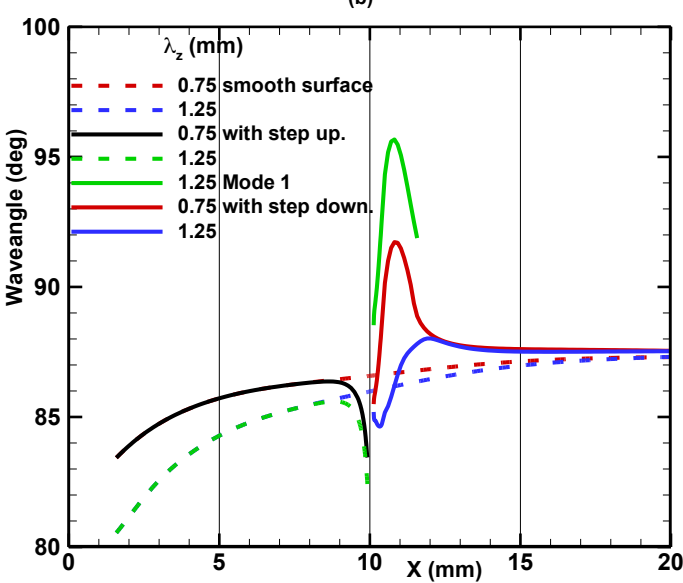

Figure 12. Variation of (a) growthrates, and (b) waveangle along the streamwise direction for stationary disturbances over a backward-facing step $\left(x_{s}=10 \mathrm{~mm}, h_{s}=0.12 \mathrm{~mm}\right)$.
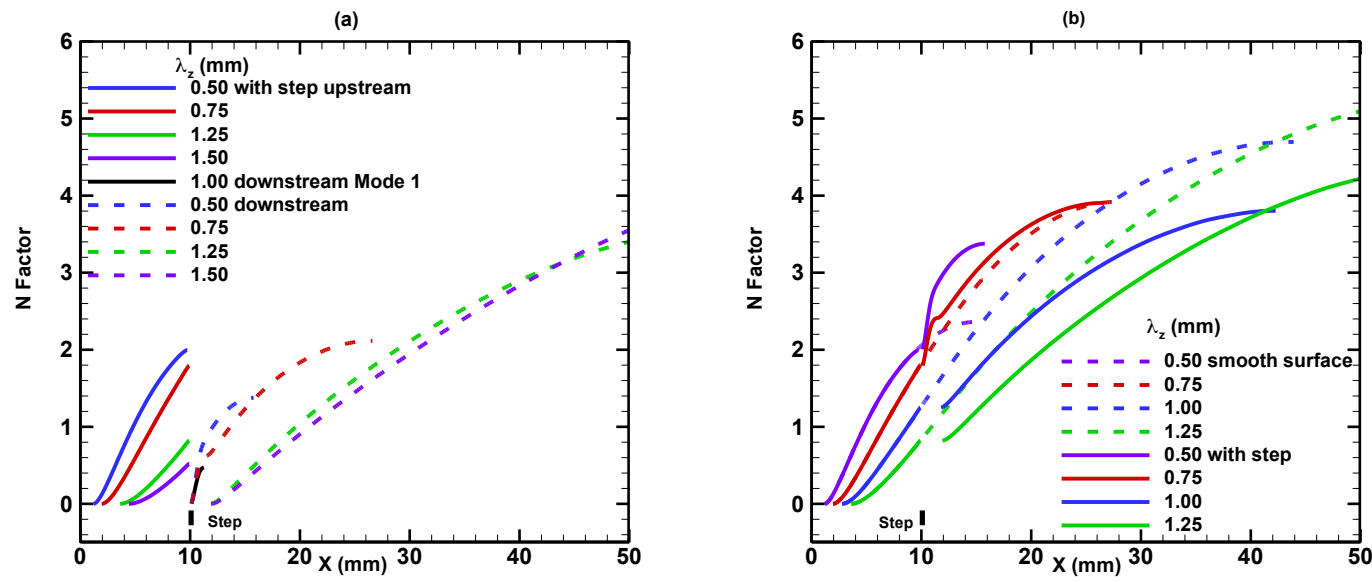

Figure 13. N-Factor for stationary disturbances with a backward-facing step ( $\left.x_{s}=10 \mathrm{~mm}, h_{s}=0.12 \mathrm{~mm}\right)$.

20 of 27

American Institute of Aeronautics and Astronautics 


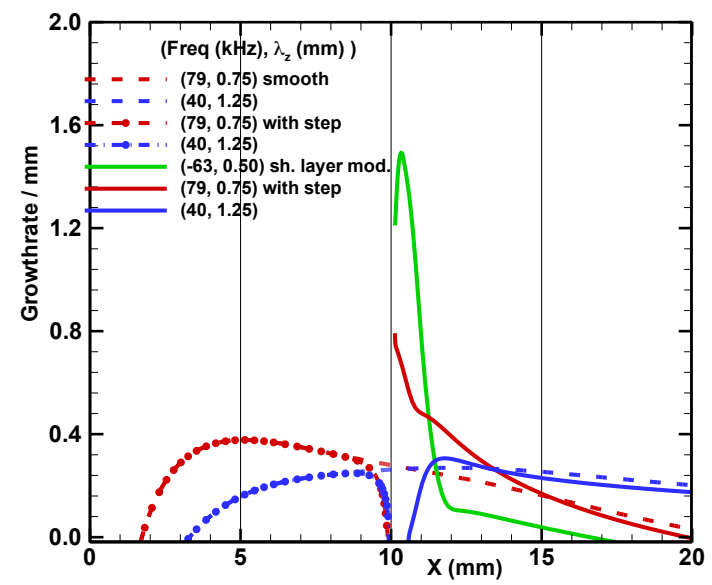

Figure 14. Variation of growthrates and waveangle along the streamwise direction for traveling disturbances for a backward-facing step $\left(x_{s}=10 \mathrm{~mm}, h_{s}=0.12 \mathrm{~mm}\right)$.
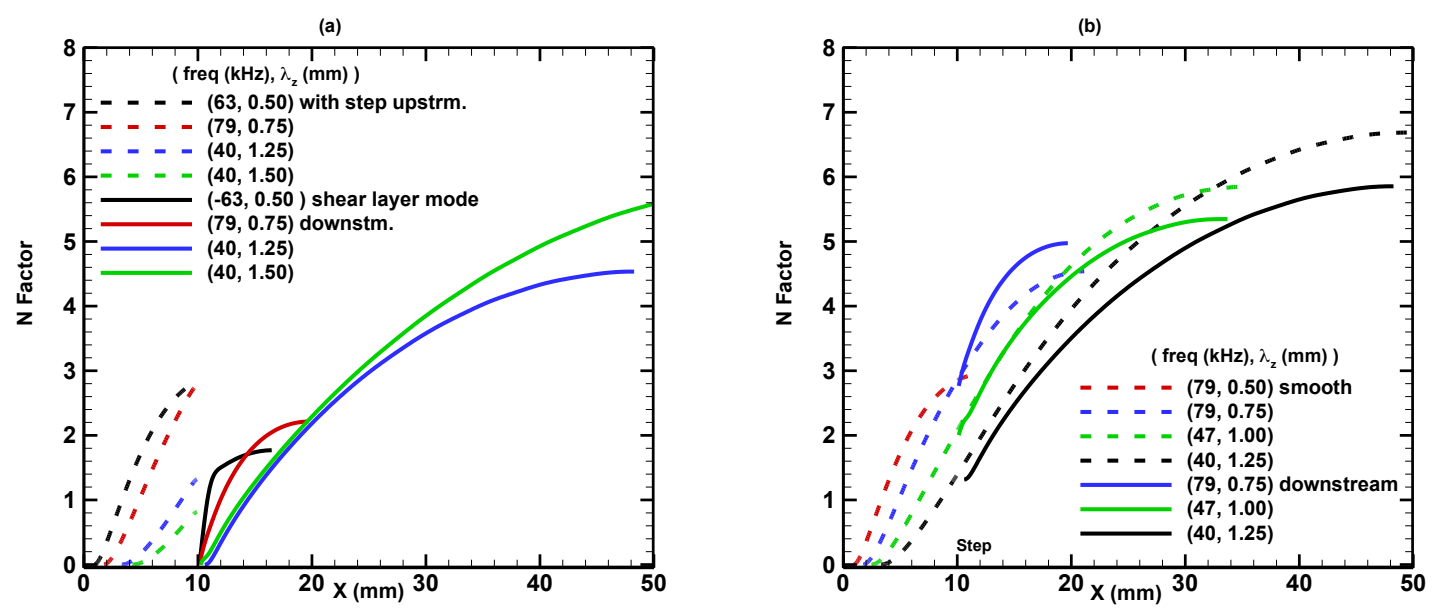

Figure 15. N-Factor variation for traveling disturbances for a backward-facing step $\left(x_{s}=10 \mathrm{~mm}, h_{s}=0.12\right.$ mm).
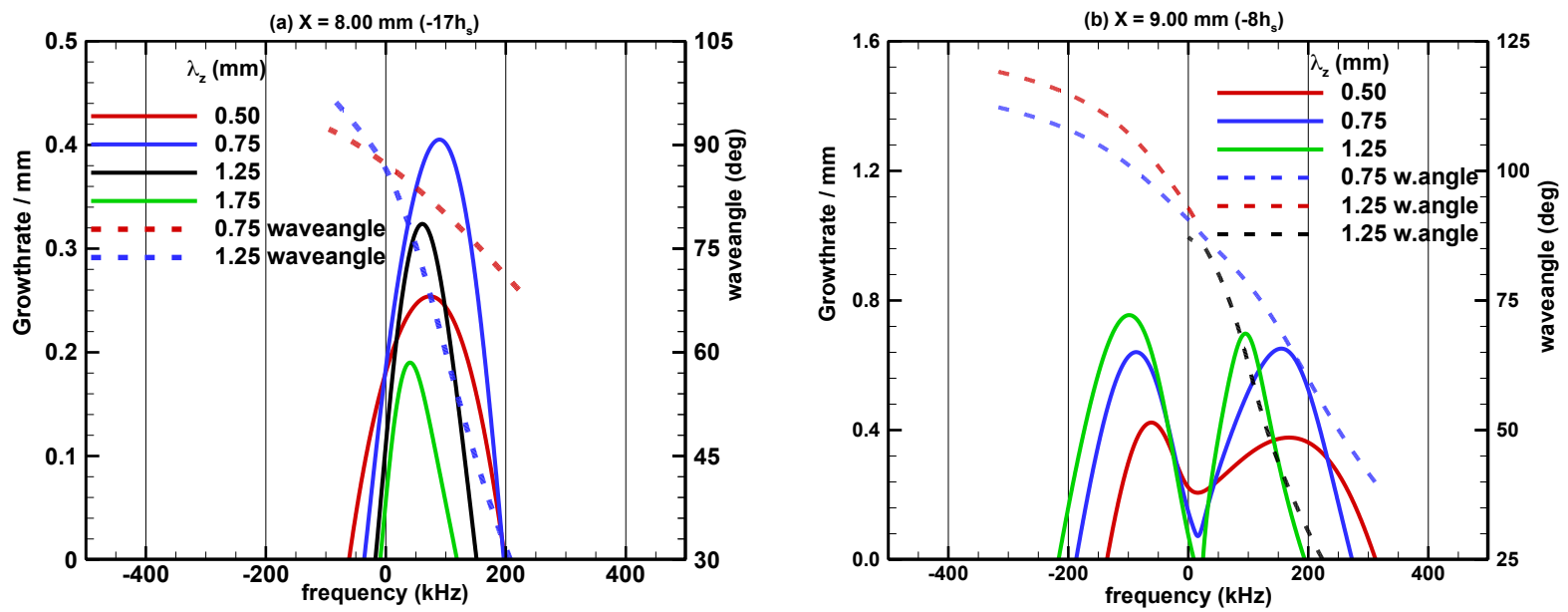

21 of 27

American Institute of Aeronautics and Astronautics 

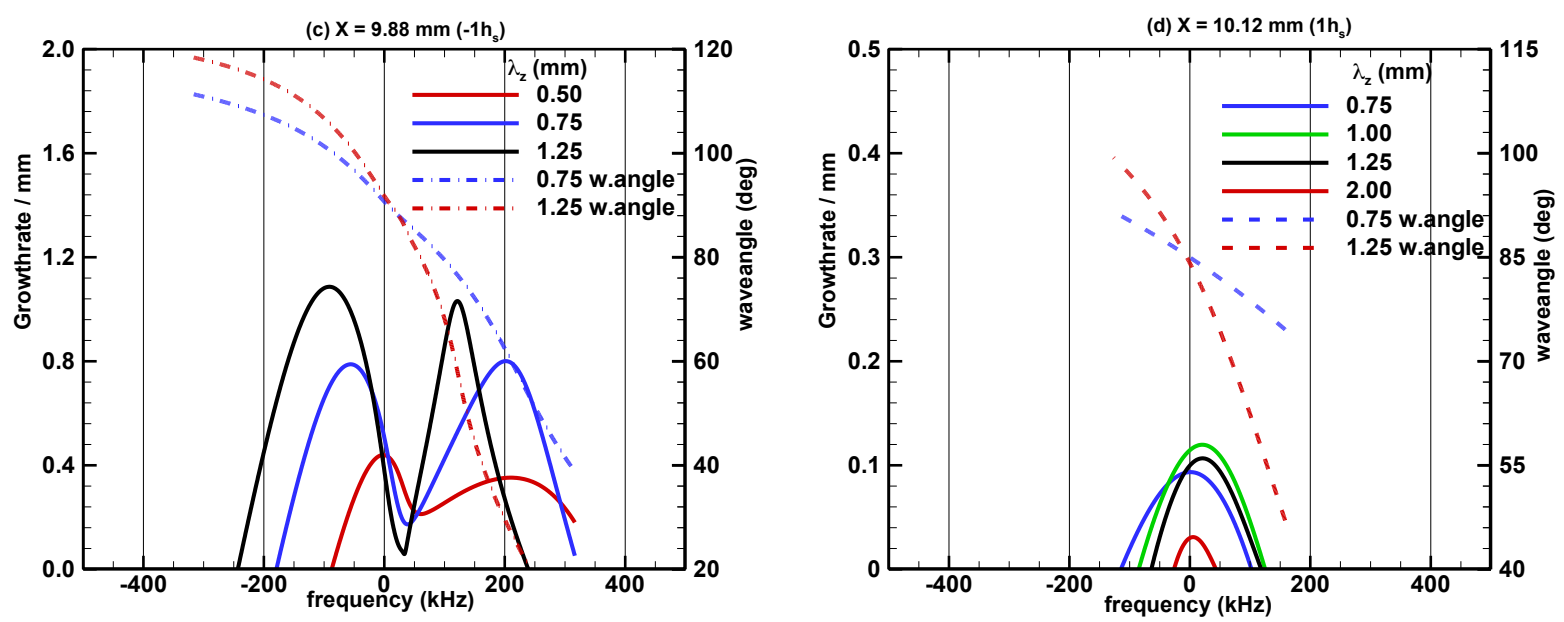

Figure 16. Variation of growthrates with the frequency for different spanwise wavelengths at upstream and downstream locations of a forward-facing step $\left(x_{s}=10 \mathrm{~mm}, h_{s}=0.12 \mathrm{~mm}\right)$.
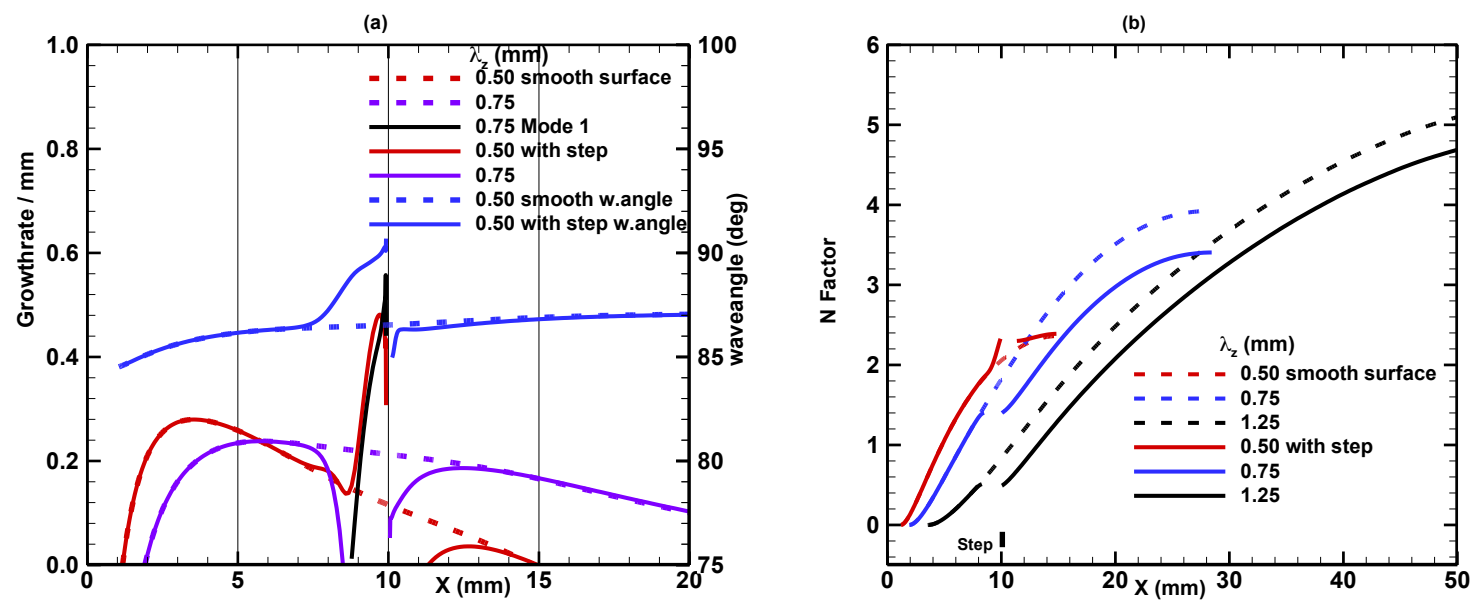

Figure 17. Variation of (a) growthrates, and (b) N-Factors along the streamwise direction for stationary disturbances over a forward-facing $\operatorname{step}\left(x_{s}=10 \mathrm{~mm}, h_{s}=0.12 \mathrm{~mm}\right)$. 
(a)

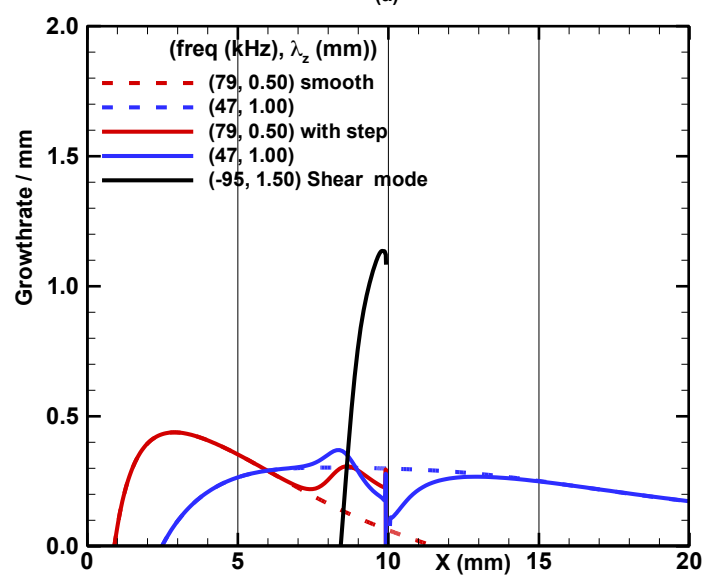

(b)

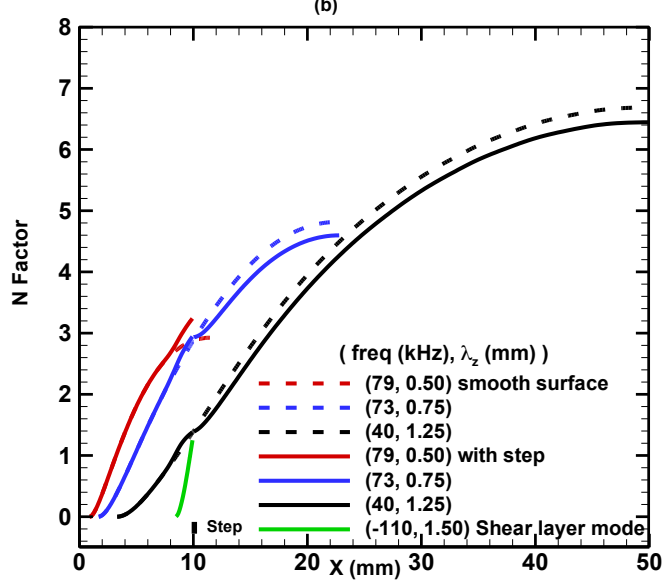

Figure 18. (a) Growthrates and (b) N-Factors computed from local stability method for traveling disturbances with a forward-facing step $\left(x_{s}=10 \mathrm{~mm}, h_{s}=0.12 \mathrm{~mm}\right)$.

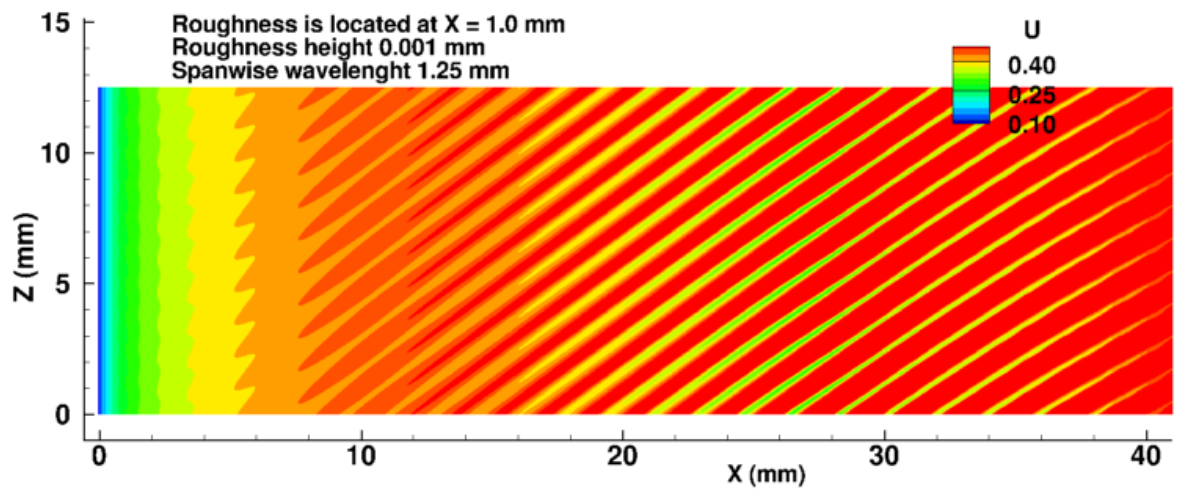

Figure 19. Contours of the $u$ velocity in the plan view $(x, z)$ plane without the step.

(a) Roughness $\lambda_{z}=1.25 \mathrm{~mm}$

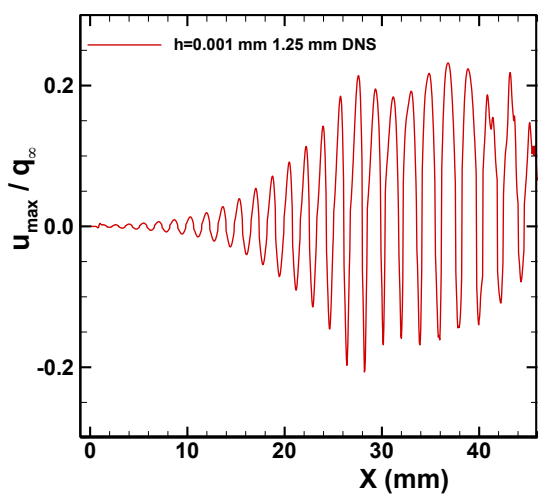

(b)

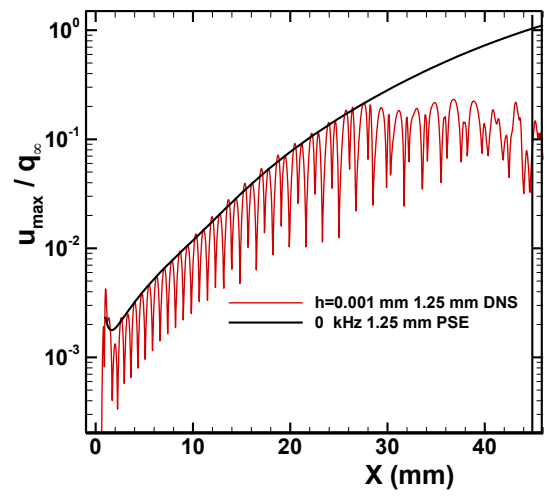

Figure 20. Perturbations of the maximum $u$-velocity component and PSE results along the blunt swept wing for perturbations generated by the roughness without the step (a) in linear scale (b) in log-scale. 

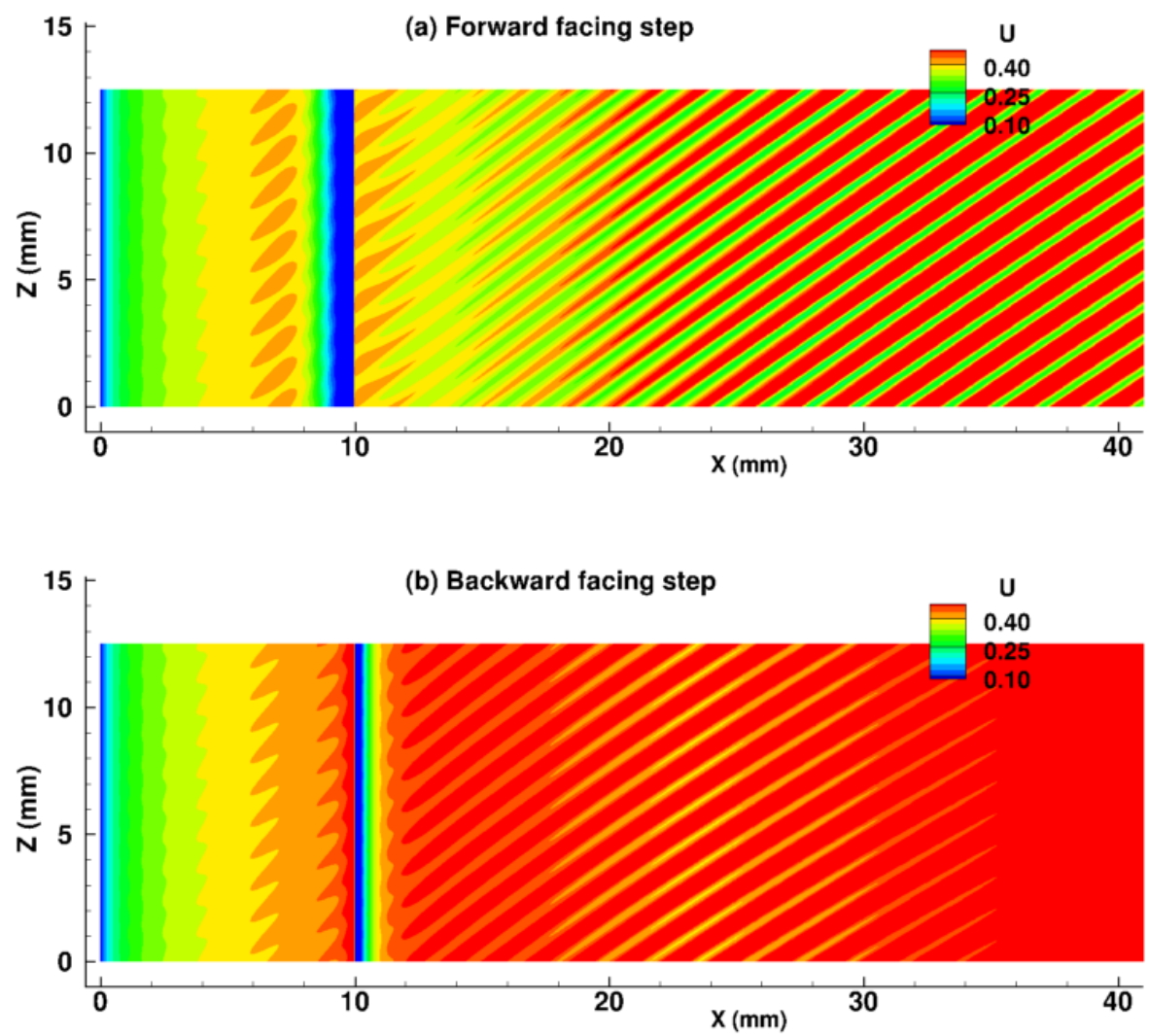

Figure 21. Contours of the $u$ velocity in the plan view $(x, z)$ plane with (a) a forward- and (b) a backwardfacing step $\left(x_{s}=10 \mathrm{~mm}, h_{s}=0.12 \mathrm{~mm}, \lambda_{\mathrm{z}}=1.25 \mathrm{~mm}\right)$. 
(a) Without step

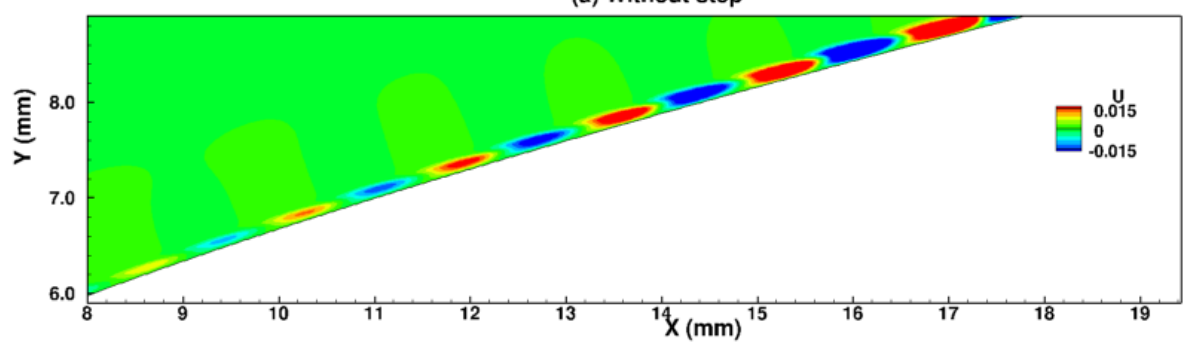

(b) Forward facing step

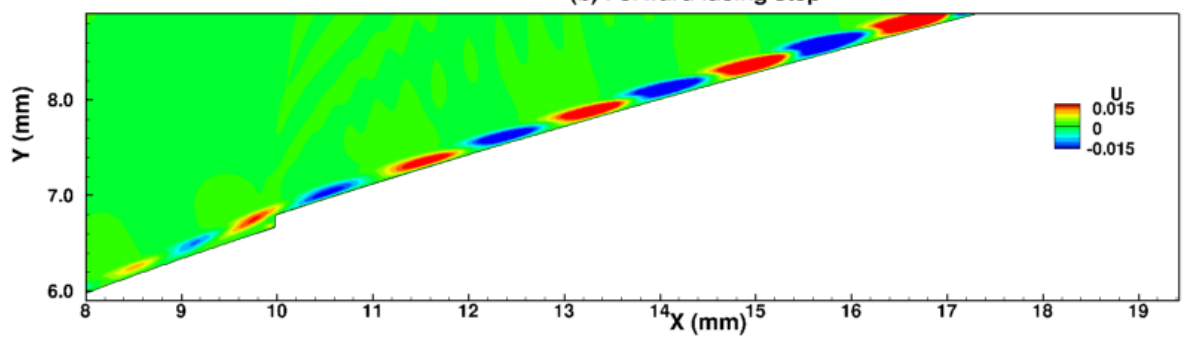

(c) Backward facing step

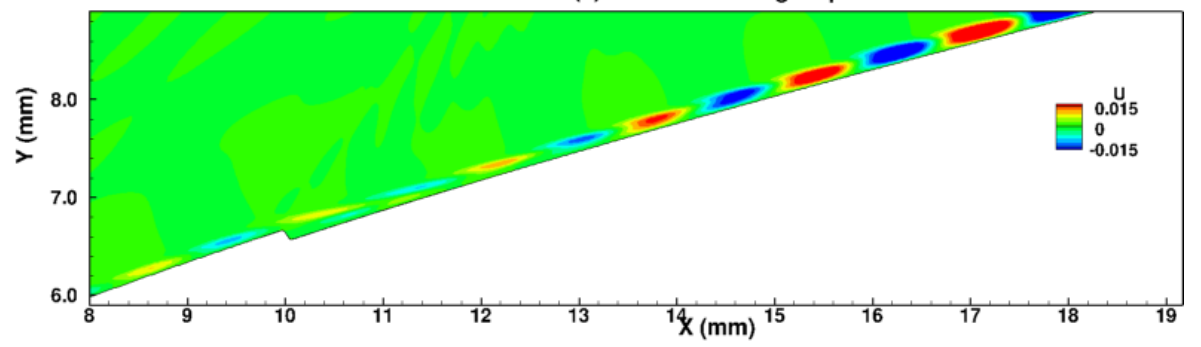

Figure 22. Contours of the $u$-velocity perturbations in the $(x, y)$ plane at $z=0$ : (a) without the step, (b) forward-facing step and (c) backward-facing step $\left(x_{s}=10 \mathrm{~mm}, h_{s}=0.12 \mathrm{~mm}, \lambda_{\mathrm{z}}=1.25 \mathrm{~mm}\right)$. 
(a) Forward facing step $h_{s}=\mathbf{0 . 0 6} \mathbf{m m}$

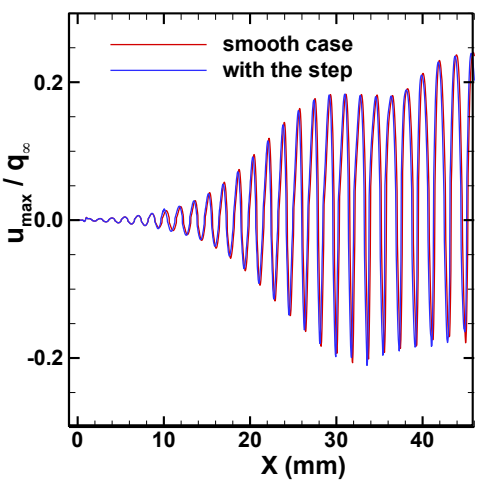

(d) Backward facing step $\mathrm{h}_{\mathrm{s}} \mathbf{= 0 . 0 6 \mathrm { mm }}$

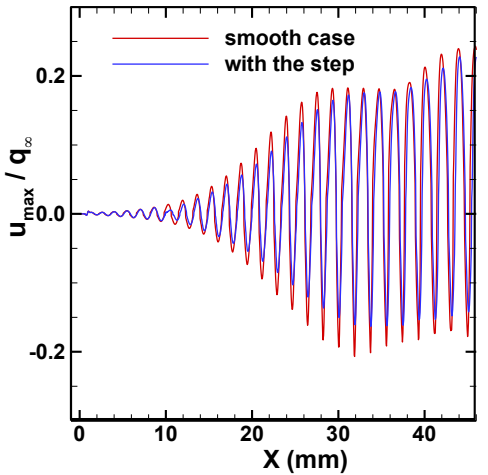

(b) Forward facing step $\mathrm{h}_{\mathrm{s}} \mathbf{=} \mathbf{0 . 1 2} \mathrm{mm}$

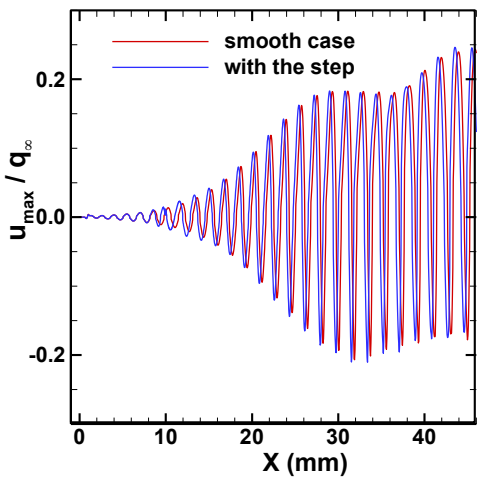

(e) Backward facing step $h_{\mathrm{s}}=\mathbf{0 . 1 2} \mathrm{mm}$

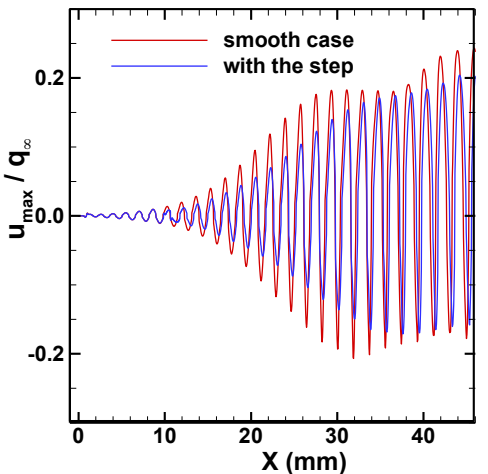

(c) Forward facing step $h_{s}=0.18 \mathrm{~mm}$

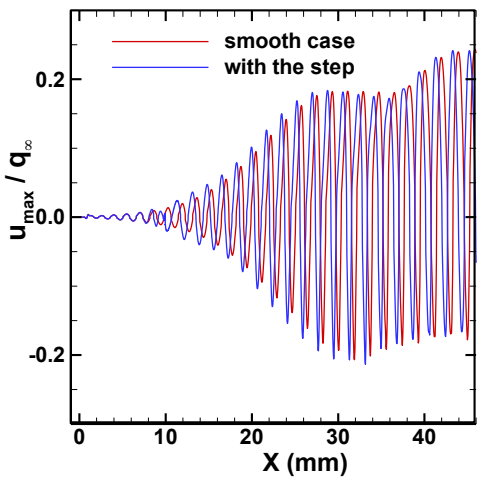

(f) Backward facing step $h_{\mathrm{s}}=\mathbf{0 . 1 8} \mathbf{m m}$

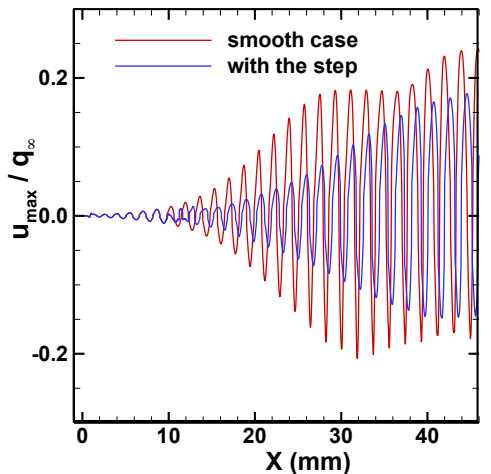

Figure 23. Perturbations of the maximum $u$-velocity component generated by the roughness with the step: (a)-(c) forward-facing step and (d)-(e) backward-facing step $\left(x_{s}=10 \mathrm{~mm}, \lambda_{\mathrm{z}}=1.25 \mathrm{~mm}\right)$.

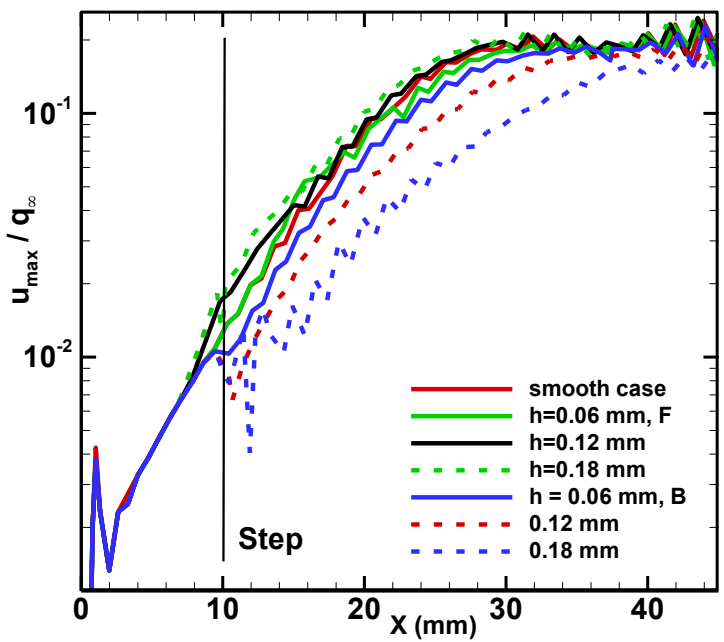

Figure 24. Perturbations of the maximum $u$-velocity component generated by the roughness with and without the $\operatorname{step}\left(x_{s}=10 \mathrm{~mm}, \lambda_{\mathrm{z}}=1.25 \mathrm{~mm}\right)$. 
(a)

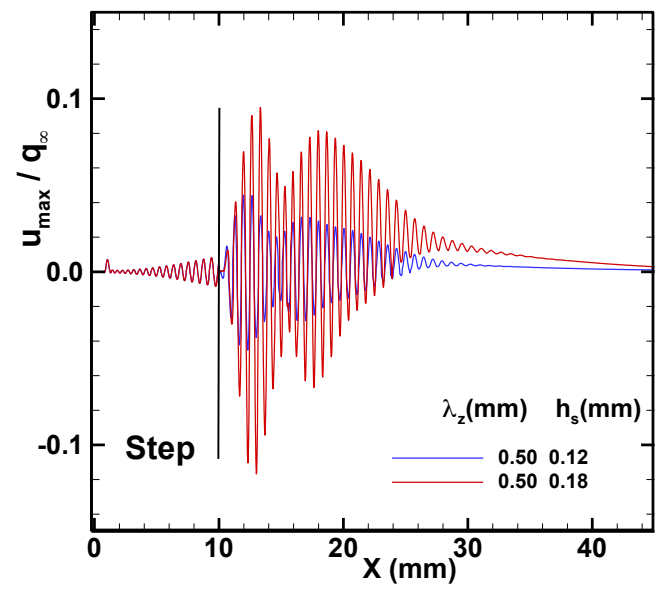

(b)

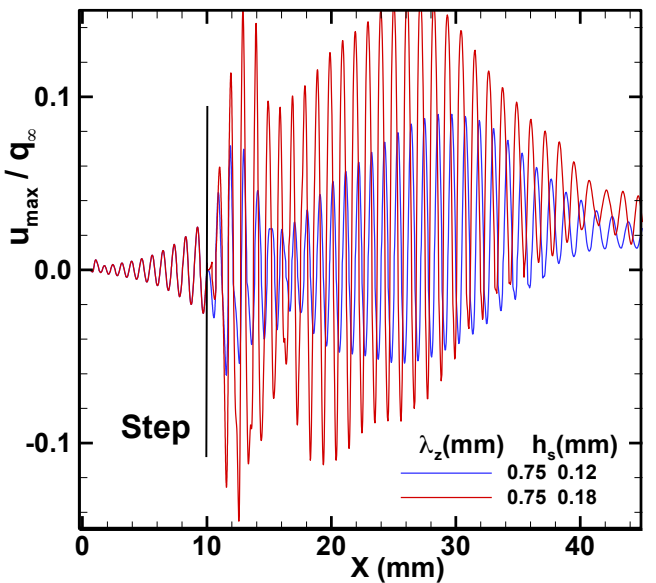

Figure 25. Perturbations of the maximum $u$-velocity component with a backward-facing step for (a) $\lambda_{z}=0.50$ $\mathrm{mm}$ and (b) $\lambda_{z}=0.75 \mathrm{~mm}$.

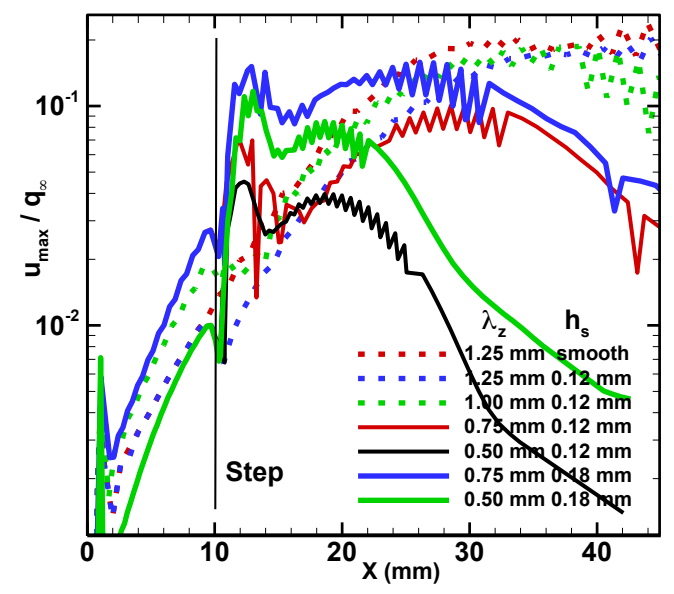

Figure 26. Maximum perturbation amplitude with a backward-facing step for different spanwise wavelengths $\left(x_{s}=10 \mathrm{~mm}\right.$ and $h_{s}=0.12$ and $\left.0.18 \mathrm{~mm}\right)$. 